\title{
Petrography and geochemistry of granitoids and related rocks from the pre-Neogene basement of the Slavonia-Srijem Depression (Croatia)
}

\author{
Sanja Šuica ${ }^{1}$, Vesnica Garašić ${ }^{2}$ and Alan B. Woodland ${ }^{3}$ \\ ${ }^{1}$ INA-Industrija nafte, d.d., Upstream Laboratory, Lovinčićeva 4, 10000, Zagreb, Croatia; (sanja.suica@ina.hr) \\ ${ }^{2}$ University of Zagreb, Faculty of Mining, Geology and Petroleum Engineering, Pierottijeva 6, 10000, Zagreb, Croatia \\ ${ }^{3}$ Goethe Universität Frankfurt, Institut für Geowissenschaften, Altenhöferallee 1, 60438 Frankfurt, Germany
}

doi: $10.4154 / g c .2022 .09$

Article history:

Manuscript received May 20, 2021

Revised manuscript accepted November 22, 2021

Available online January 31,2022

\begin{abstract}
The pre-Neogene basement of the Slavonia-Srijem Depression (eastern Croatia) is composed of various types of igneous, metamorphic and sedimentary rocks. Here we present the petrography and geochemistry of a heterogenous group represented by two types of alkali granite, granite, syenite, rhyolite and orthogneiss. The alkali granite type 1 has an A-type geochemical affinity: a ferroan character, high alkali content, high concentration of rare earth elements $\left(\mathrm{REE}^{3+}\right)$, $\mathrm{Rb}, \mathrm{Zr}, \mathrm{Nb}$ and $\mathrm{Y}$, and low $\mathrm{CaO}, \mathrm{MgO}, \mathrm{P}_{2} \mathrm{O}_{5}, \mathrm{Ba}$, Sr and Eu contents. The syenite has similar characteristics, but displays enrichment in $\mathrm{Ba}, \mathrm{K}$, Eu and $\mathrm{Zr}$, which could be a consequence of feldspar and zircon accumulation. The alkali granite type 2 is an A-type granite but differs from the alkali granite type 1 in having lower $\mathrm{K}_{2} \mathrm{O}$ and $\mathrm{Rb}$, accompanied by higher $\mathrm{Na}_{2} \mathrm{O}$ and $\mathrm{Sr}$ concentrations, possibly resulting from alteration or a different parental magma/evolutionary process. The granite and rhyolite are distinguished from both types of alkali granite by their magnesian character, lower $\mathrm{Zr}, \mathrm{Nb}$ and $\mathrm{Y}$ concentrations, less pronounced Eu negative anomaly, as well as higher $\mathrm{Ba}$, $\mathrm{Sr}$ and LREE/HREE. The orthogneiss displays differences in major element chemistry compared to the alkali granite type 1, but has similar trace element and REE patterns. The alkali granites are characterized by $\mathrm{Y} / \mathrm{Nb}<1.2$, indicating an ocean island basalt-like source, while the granite originated from melting of a crustal, probably metasedimentary source. The Atype granites could belong to the Late Cretaceous A-type magmatism of the Sava Zone, while the granite is significantly different from the Sava Zone A-type granites as well as the other rocks investigated in this study.
\end{abstract}

Keywords: A-type granite, pre-Neogene basement, Slavonia-Srijem Depression, Sava Zone reports the results of petrography and whole-rock geochemistry for two types of alkali granite, granite, syenite, rhyolite and orthogneiss as well as the mineral chemistry for alkali granite type 1 . The aim of this study was to define granitoid rocks in the preNeogene basement of the SSD, obtain information about their sources, potential genetic relationships and suggest the regional context of the examined rocks.

\section{GEOLOGICAL SETTING}

The Sava Zone is defined as a relatively narrow belt composed of various types of igneous and metamorphic rocks along with the Late Cretaceous sedimentary rocks. It is interpreted as a suture zone between the European plate (Tisia-Dacia) and the Adriatic plate, stretching E-W from Zagreb to Belgrade and NNW-SSE along the Vardar River to the Aegean Sea (Fig. 1; SCHMID et al., 2008, 2020; USTASZEWSKI et al., 2010). According to SCHMID et al. (2008), the north-western tip of the Sava Zone takes on a SW-NE strike, following the Mid-Hungarian Fault Zone. USTASZEWSKI et al. (2009, 2010) interpreted the Sava Zone as an ocean basin, a relic of the Mesozoic Tethys (Vardar Ocean) that remained open during the Late Cretaceous and Early Palaeogene. According to GALLHOFER et al. (2015) the subduction of this oceanic lithosphere led to establishment of the Late Cretaceous magmatic arc of the Apuseni-Banat-Timok-Srednogorie Belt.

One of the most distinctive features of the Sava Zone is the Late Cretaceous bimodal magmatism, manifested predominantly by basic and acidic volcanism with minor shallow plutonism (e.g. acidic rocks from samples obtained from boreholes. This paper 


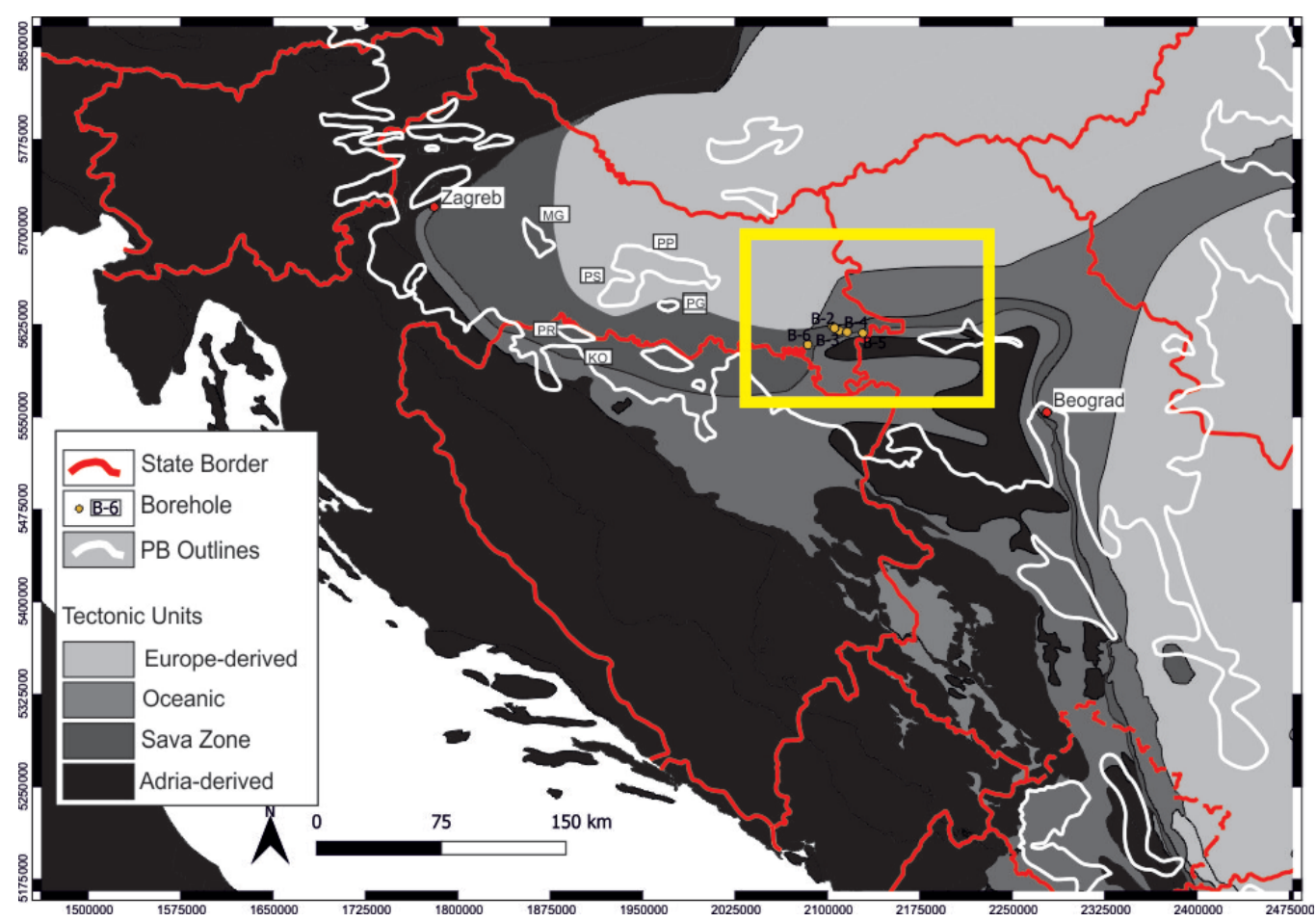

Figure 1. Regional map of the major tectonic units simplified after SCHMID et al. (2020). Europe-derived units include the Tisia and Dacia megaunits, while the Adria-derived units include the Adriatic microplate, deformed Adriatic margin and Adria-derived thrust sheets. Oceanic units are the Neotethyan obducted ophiolites. The yellow rectangle denotes the position of the study area depicted in Fig. 2. MG: Moslavačka Gora, PP: Papuk, PS: Psunj, PG: Požeška Gora, PR: Prosara, KO: Kozara.

PAMIĆ, 2002), outcropping on the Požeška Gora in Croatia (ŠPARICA \& PAMIĆ, 1986; PAMIĆ et al., 1988, 1988/1989), the Kozara (USTASZEWSKI et al., 2009; CVETKOVIĆ et al., 2014) and the Prosara (PAMIĆ \& INJUK, 1988; USTASZEWSKI et al., 2010) in Bosnia and Herzegovina and the Klepa in North Macedonia (PRELEVIĆ et al., 2017). This bimodal magmatism covers the time span of 83.6-81 Ma(USTASZEWSKI et al., 2009; BALEN et al., 2020). The geodynamic context of this magmatism varies between different authors: it is possibly related to a backarc basin (PAMIĆ, 2002; USTASZEWSKI et al., 2009), or an anomalous Iceland-type mid-oceanic ridge (CVETKOVIĆ et al., 2014), or a continental rift/pseudo-rift (BELAK et al., 1998), or an upper-plate fore-arc basin (PRELEVIĆ et al., 2017), or due to intracontinental extension induced by transtensional tectonics (PRELEVIĆ et al., 2017). The acidic magmatism itself also has various interpretations: from a product of partial melting of basic oceanic crust on the Kozara (CVETKOVIĆ et al., 2014), or a product of basic melt fractionation, along with minor crustal contamination on the Požeška Gora (PAMIĆ et al., 2000). The Atype granite of the Požeška Gora is considered to be a product of partial melting of the continental crust, along with a possible mantle contribution (BALEN et al., 2020). Besides this bimodal volcanism, Late Cretaceous ultrapotassic lamprophyric volcanism was recently described at the Ripanj locality, near Belgrade, Serbia by SOKOL et al. (2020). They attributed this volcanism to melts derived from the partial melting of metasomatized lithospheric mantle either in a fore-arc or an intracontinental setting associated with transtensional tectonics.

The eastern part of the SSD is completely covered by Quaternary deposits (BRKIĆ et al., 1989; ČIČULIĆ-TRIFUNOVIĆ \& GALOVIĆ, 1984). According to the regional interpretations of SCHMID et al. $(2008,2020)$, the pre-Neogene basement of the SSD includes Adria-derived tectonic units, the Sava Zone and
Europe-derived units (Fig. 2). It is mainly composed of igneous (diabase, gabbro, granite, syenite) and metamorphic (gneiss and mica schist, low grade metasediments) rocks, while sedimentary rocks such as flysch-like sandstones and shales are subordinate. Based on the results of whole rock K-Ar ages, PAMIĆ \& PÉCSKAY (1994) considered basic rocks from the SSD to comprise part of a Late Cretaceous ophiolite sequence. The granite and gneiss, along with low grade metamorphic rocks have been correlated with the Hercynian complex of the Papuk Mts. (PAMIĆ \& LANPHERE 1991).

\section{SAMPLING AND ANALYTICAL METHODS}

A brief overview on the core samples along with the borehole number, depth interval and type of analysis is provided in Table 1. The borehole data was obtained from INA-Industrija nafte d.d. well reports. In the studied boreholes, the basement is composed of alkali granite type 1 (B-1 borehole, apparent thickness $>205 \mathrm{~m}$ ), alkali granite type 2 (B-2 borehole, apparent thickness $>98 \mathrm{~m}$ ), syenite (B-3 borehole, apparent thickness $>61 \mathrm{~m}$ ), orthogneiss (B-4 and B-5 boreholes, apparent thickness $>57 \mathrm{~m}$ and $308 \mathrm{~m}$, respectively) and cataclastic porphyritic granitic breccia with rhyolite fragment (B-6 borehole, apparent thickness of $489 \mathrm{~m}$ ). A rhyolite fragment was sampled along with the granite in order to test whether there is any cogenetic relationship between these two types of rocks. The orthogneiss from B-4 and B-5 boreholes was sampled for the same reason, i.e. to check for a possible relationship with the adjacent granites. The sampled rocks are covered by Late Miocene marls, except in B-5, where Early-Middle Miocene breccia-conglomerates are present. The only borehole that was drilled through the studied units was B-6, where flysch-like interbedded sandstone and shale underlie the granitic breccia. In total, nine samples were investigated from six boreholes. Petrographic analysis was done using Leitz Wetzlar Orthoplan and 


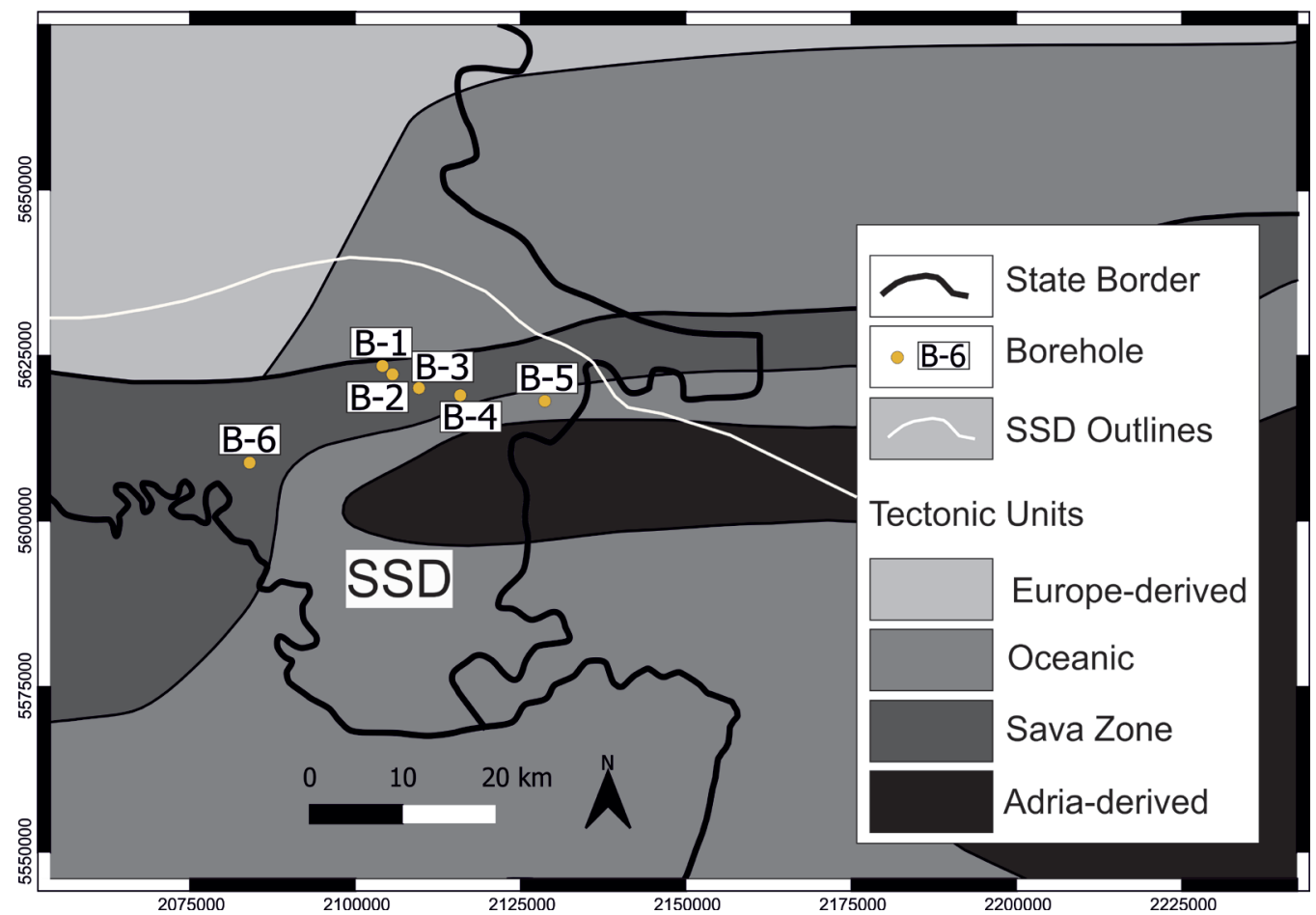

Figure 2. Map of the major tectonic units of the study area with an approximate outline of the Slavonia-Srijem (SS) Depression, along with the position of the boreholes from which the samples were obtained. Simplified after SCHMID et al. (2020).

Table 1. List of samples with borehole number, depth interval and type of analysis. WRA - whole rock analysis. EMPA - mineral chemistry by electron probe micro-analyser.

\begin{tabular}{lllll}
\hline Sample & Borehole & Depth interval $(\mathrm{m})$ & Analysis & Rock type \\
\hline B-1-3 & B-1 & $1010-1011$ & WRA & alkali granite type 1 \\
\hline B-1-4 & B-1 & $1070-1072$ & WRA & alkali granite type 1 \\
\hline B-1-5 & B-1 & $1140-1141.5$ & WRA, EMPA & alkali granite type 1 \\
\hline B-2-1 & B-2 & $1028-1029.5$ & WRA & alkali granite type 2 \\
\hline B-3-5 & B-3 & $1093-1099$ & WRA & syenite \\
\hline B-4-5 & B-4 & $1156-1158.5$ & WRA & orthogneiss \\
\hline B-5-2 & B-5 & $1568-1570$ & WRA & orthogneiss \\
\hline B-6-2 & B-6 & $1427-1430$ & WRA & granite \\
\hline B-6-2 & B-6 & $1427-1430$ & WRA & rhyolite \\
\hline
\end{tabular}

Olympus BX51 polarizing microscopes at the Upstream Laboratory, INA-Industija nafte, d.d., Zagreb. Modal analysis was obtained by point-counting of at least 500 points.

The mineral chemistry for one sample was obtained with a JEOL JXA 8900 electron probe micro-analyser (EPMA) at the Institut für Geowissenschaften, Goethe Universität Frankfurt. Measurements on a carbon-coated thin section and polished grain mount were performed in wavelength-dispersive mode (WDS) with a $15 \mathrm{kV}$ accelerating voltage, a $20 \mathrm{nA}$ (12 nA for feldspars) beam current and $\sim 3 \mu \mathrm{m}$ beam diameter. Raw data were corrected with the CITZAF quantitative matrix correction implemented by JEOL.

The whole rock chemical analysis was performed by inductively coupled plasma emission spectroscopy (ICP-ES) for major elements, carbon and sulphur, while trace elements including rare-earth elements (REE) were determined by inductively coupled plasma mass spectrometry (ICP-MS), at the Bureau Veritas
Minerals, Vancouver, Canada. The rocks were analysed after sample solution by 4 -acid digestion of $0.25 \mathrm{~g}$ sample, while the REE were determined after $\mathrm{LiBO}_{2} / \mathrm{Li}_{2} \mathrm{~B}_{4} \mathrm{O}_{7}$ fusion and nitric acid digestion of a $0.1 \mathrm{~g}$ sample. Detection limits for ICP-ES analyses was $0.01 \%$, except for $\mathrm{SiO}_{2}(0.04 \%), \mathrm{Al}_{2} \mathrm{O}_{3}(0.03 \%)$ and $\mathrm{Fe}_{2} \mathrm{O}_{3}$ (0.04\%), while detection limits for ICP-MS analysis were 0.1 ppm. GCDkit 4.1 (JANOUŠEK et al., 2006) was used for data handling.

\section{RESULTS}

The granitoid rocks analysed in this study are classified based on geochemical characteristics, using the classification of DE LA ROCHE et al. (1980; Fig. 3). The main reason for using this classification instead of the quartz-alkali feldspar-plagioclase (QAP)

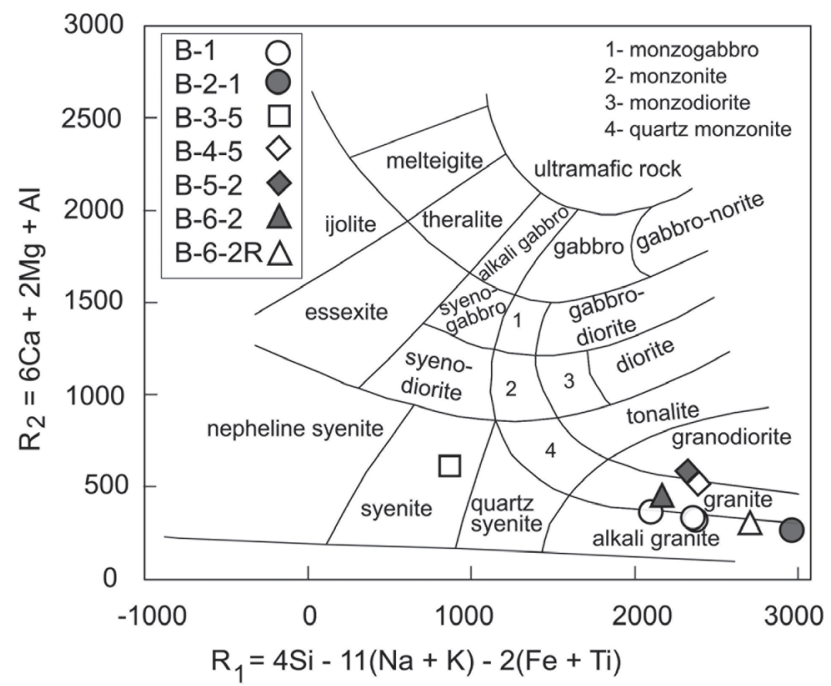

Figure 3. Classification diagram based on major elements after DE LA ROCHE et al. (1980). 
classification of STRECKEISEN (1974) based on modal composition is the lack of mineral chemistry data for all the samples. Consequently, we have not made the distinction between albite with $<5 \%$ Ab component, which is according to STRECKEISEN (1974), included in the alkali feldspar group and albite with $>5 \%$ Ab which is included in the plagioclase group. Rhyolite is classified according to major element composition, i.e. total alkali-silica diagram (LE BAS et al, 1986; not displayed).

\subsection{Petrography}

The alkali granite type 1 (samples B-1-3, B-1-4, B-1-5) is a pinkcoloured, leucocratic, medium-grained rock with an equigranular texture (Fig. 4a) and is locally porphyritic. The rock is composed of K-feldspar (58-67\%) and quartz (25-37\%) followed by minor albite (2-6\%), accessory biotite and hornblende, magnetite, ilmenite, zircon and apatite. The subsolidus mineral assemblage includes chlorite (1-2\%), stilpnomelane, allanite, epidote, fluorite, titanite, haematite, pyrite, calcite, sericite and kaolinite. Anhedral $K$-feldspar (up to $5 \mathrm{~mm}$ ) has perthitic texture with irregular albite lamellae $(20-50 \%$ of the host mineral). It forms graphic intergrowths with quartz, which is especially pronounced in the finegrained matrix of the porphyritic varieties, where these intergrowths are irregular and of different size. Quartz (up to $3 \mathrm{~mm}$ ) is anhedral, with undulose extinction. Prismatic albite (up to 2
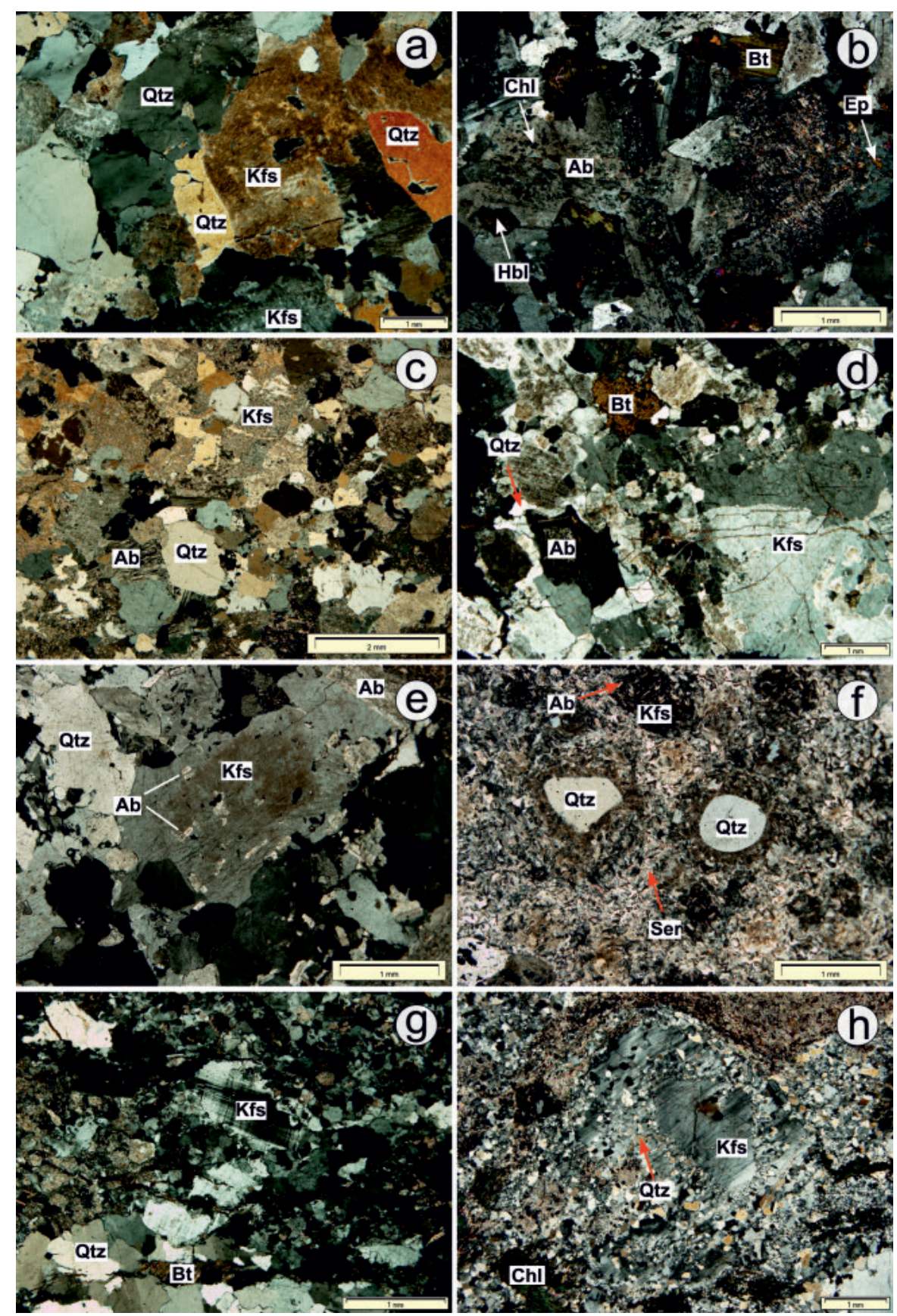

Figure 4. Photomicrographs of: a) K-feldspar (Kfs) and quartz (Qtz) in equigranular alkali granite type 1 (B-1-5); b) clusters of albite (Ab), biotite (Bt) and hornblende $(\mathrm{Hbl})$ with chlorite (Chl) and epidote (Ep) replacing albite in alkali granite type 1 (B-1-5); C) albite (Ab), sericitized K-feldspar (Kfs) and quartz (Qtz) in alkali granite type 2 (B-2-1); d) K-feldspar (Kfs), albite (Ab) and quartz (Qtz) in syenite (B-3-5), interstitial biotite (Bt); e) granite (B-6-2) with quartz (Qtz) and K-feldspar (Kfs) containing albite (Ab) inclusions; f) rounded quartz (Qtz) phenocrysts, K-feldspar (Kfs) with albite (Ab) inclusions, sericite (Ser) in groundmass of rhyolite (B-6-2R); $\mathrm{g}$ ) Kfeldspar (Kfs) microcline (Mc), quartz (Qtz) and biotite (Bt) in gneiss (B-5-2); h) K-feldspar (Kfs) porphyroclast mantled by quartz (Qtz) and secondary chlorite (Chl) in gneiss (B-4-5). Abbreviations of mineral names after WHITNEY \& EVANS (2010). 
$\mathrm{mm})$, with thin polysynthetic twining, is associated with rounded clusters of hornblende and biotite (Fig. 4b), where it is characterized by altered cores containing different proportions of small epidote grains and/or chlorite aggregates, usually surrounded by fresh albite rims. Biotite (up to $2 \mathrm{~mm}$ ) is the most abundant mafic mineral and it occurs as anhedral flakes, with light to dark brown pleochroism. It is mainly interstitial to feldspar and quartz. Hornblende $(<1 \mathrm{~mm})$ is euhedral to subhedral, pleochroic from light to dark green and is mainly altered to stilpnomelane and minor chlorite, along with epidote, titanite and opaques.

The alkali granite type 2 (B-2-1) is a grey to pinkish, leucocratic, fine-grained $(<1.5 \mathrm{~mm})$ rock, characterized by pronounced quartz-feldspar graphic intergrowths. It generally has an equigranular texture (Fig. $4 \mathrm{c}$ ) but is locally porphyritic. The rock is composed of quartz (46\%), albite (40\%) and K-feldspar (8\%), followed by accessory apatite, zircon and opaques. Subsolidus minerals include sericite (6\%), chlorite, haematite, carbonate and kaolinite. Feldspars and quartz (to a lesser extent) occur as larger grains, and in the granophyric groundmass of the porphyritic variety. They are extensively altered to sericite. Albite locally has an antiperthitic texture, with K-feldspar exsolution lamellae, and a subordinately altered core with fresh rim. $K$-feldspar is perthitic. The primary ferromagnesian mineral has been completely altered to chlorite. Chlorite and sericite also occur interstitially as fan-shaped aggregates.

The syenite (B-3-5) is a pinkish, leucocratic, coarse-grained rock, displaying a cataclastic and locally granular texture. The rock is composed of K-feldspar (56\%) and albite (32\%), followed by minor quartz (5\%), biotite $(4 \%)$, and hornblende $(2 \%)$. Accessory minerals are zircon, apatite and opaques. Subsolidus minerals are chlorite, epidote, haematite, carbonate, sericite and kaolinite. Microstructures indicative of the plastic deformation were observed: e.g. larger grains of K-feldspar surrounded by myrmekite, distortion of polysynthetic twining in albite and kink-bands in biotite laths. $K$-feldspar (up to $30 \mathrm{~mm}$ ) has an anhedral habit, perthitic texture, and contains inclusions of albite. Albite (up to $10 \mathrm{~mm}$ ) is anhedral and displays thin polysynthetic twinning. It occurs predominantly with narrow fresh rims around altered grains. Quartz is anhedral, interstitial, and up to $0.5 \mathrm{~mm}$ in size (Fig. 4d). Biotite (up to $2 \mathrm{~mm}$ ) is interstitial, with light to dark brown pleochroism. Hornblende (up to $2 \mathrm{~mm}$ ) is subhedral and pleochroic from light to dark green.

The granite (B-6-2) is leucocratic rock, with red K-feldspar, pinkish-orange albite and greyish quartz, displaying seriate porphyritic texture (Fig. 4e). K-feldspar megacrysts occur locally. The texture is locally cataclastic, with fragments of granite in a dark green matrix, and shear zones up to $1 \mathrm{~cm}$ wide. The rock is composed of albite (37\%), K-feldspar (31\%) and quartz (26\%). Accessory minerals are zircon, apatite and opaques. Subsolidus minerals are chlorite $(6 \%)$, white mica, clay minerals and carbonate. $K$-feldspar $(0.1$ to $>50 \mathrm{~mm})$ has an anhedral habit and perthitic texture with thin albite exsolution. Larger grains of K-feldspars contain inclusions of albite microliths and quartz grains. K-feldspar megacrysts are anhedral, without sharp boundaries with surrounding matrix and contain zonally distributed quartz, albite and chlorite inclusions (up to $0.5 \mathrm{~mm}$ in size). These large grains also exhibit cracks filled with subsolidus quartz or carbonate. Albite ( 0.1 to $15 \mathrm{~mm}$ ) has an anhedral to subhedral habit and thin polysynthetic twining. Larger albite grains contain K-feldspar and quartz inclusions. Quartz (up to $10 \mathrm{~mm}$ ) is anhedral, displaying segmentation, undulose extinction and deformation lamellae. Larger quartz grains contain albite, K-feldspar and chlo- rite inclusions. Chlorite (0.1-1.5 mm) displays light green to green pleochroism. It occurs as pseudomorphs after primary ferromagnesian minerals, probably biotite, with opaque inclusions and locally developed kink-band structures. It also occurs as interstitial and fracture filling fine-grained aggregates.

The rhyolite (B-6-2R) is a light olive grey rock with aphyric texture and a phenocryst to matrix ratio of 1:40. It contains few phenocrysts of quartz and subordinate feldspar in a devitrified spherulitic and micropoikilitic groundmass (Fig. 4f). Quartz phenocrysts $(\sim 0.5 \mathrm{~mm})$ are rounded, sub rounded, subhedral and partially embayed. Quartz is also a part of the groundmass, filling cuspate areas between spherulites. Feldspar phenocrysts (up to $1.1 \mathrm{~mm}$ ) are subhedral, with a prismatic habit and are almost completely altered to sericite and clay minerals. The groundmass is composed of quartz, feldspar and secondary white mica.

The orthogneiss (B-5-2, B-4-5), with porphyroclastic texture, contains pinkish feldspar porphyroclasts in greyish green matrix. The foliated fabric is defined by the alternation of quartz and/or quartz-feldspar ribbons and strips of parallel-oriented mica. The rock is composed of quartz (35-45\%), albite (20-28\%), K-feldspar (13-25\%), muscovite (5-12\%), biotite (6\%), garnet (1\%) and local clinozoisite (0-3\%). Accessory minerals are zircon, apatite and opaque minerals, along with local tourmaline. Secondary minerals are chlorite, sericite, carbonate, haematite, pyrite and clay minerals. Quartz ( 0.05 to $2 \mathrm{~mm}$ ) is anhedral, displaying subgrains or undulose extinction, mainly forming monomineralic aggregates. Larger quartz grains, locally mantled by fine-grained quartz aggregates, display segmentation to prismatic subgrains. $K$-feldspar (0.1 to $20 \mathrm{~mm}$ ) is anhedral, locally with tartan twinning (Fig. 4 g). It is mainly associated with quartz-rich domains. Larger K-feldspar grains are perthitic, locally surrounded by finegrained aggregates of quartz and subordinately plagioclase, contain fractures and display undulose extinction (Fig. 4h). Albite (0.1 to $2 \mathrm{~mm}$ ) has an anhedral habit and thin polysynthetic twining, which is locally deformed. Biotite ( 0.1 to $1 \mathrm{~mm}$ ) has an anhedral habit and light to dark brown pleochroism. It occurs in quartz domains and as inclusions inside larger K-feldspar grains and locally forms lenticular clusters. Muscovite ( 0.1 to $0.6 \mathrm{~mm}$ ) is anhedral to subhedral and parallel-oriented, forming the strips. Garnet $(0.1-1 \mathrm{~mm})$ is anhedral to subhedral and mainly atollshaped. Clinozoisite (up to $1 \mathrm{~mm}$ ), occuring only in sample B-45 , displays a preferred orientation of elongate grains.

\subsection{Mineral chemistry}

The sample of the alkali granite type 1 (B-1-5) was selected for mineral chemical analysis based on petrographic observations. The rest of the samples have not been subjected to the analysis of mineral chemistry due to alteration. The analysed minerals were amphibole, biotite and feldspar.

The results of the selected EPMA analyses of amphibole from the alkali granite type 1 (B-1-5) are listed in Table 2. The structural formula of amphibole was calculated based on 23 oxygen atoms and 15 cations. According to LEAKE et al. (1997), the analysed amphibole is calcic with $\mathrm{Ca}_{\mathrm{B}}>1.5$ atom per formula unit (apfu), corresponding to ferro-edenite (Fig. 5), with $(\mathrm{Na}+\mathrm{K})_{\mathrm{A}}=$ 0.54-0.75 apfu. The $\mathrm{Fe} /(\mathrm{Fe}+\mathrm{Mg})$ ratios range from 0.79 to 0.85 , while total aluminium $\mathrm{Al}_{\text {tot }}$ is low (1.08-1.34 apfu).

The results of the selected EPMA analyses of biotite are provided in Table 3 . The structural formula of biotite was calculated based on 24 atoms $(\mathrm{O}, \mathrm{OH})$ and 15 cations. The analysed biotite corresponds to annite, with $\mathrm{Fe} /(\mathrm{Fe}+\mathrm{Mg})$ ratio ranging from 0.66 
Table 2. Selected EPMA analyses and structural formulae of amphibole from the alkali granite type 1 (B-1-5). Calculated based on 23 oxygen atoms and 15 cations.

\begin{tabular}{|c|c|c|c|c|c|c|c|c|c|c|c|c|}
\hline Sample & B-1-5 & B-1-5 & B-1-5 & B-1-5 & B-1-5 & B-1-5 & B-1-5 & B-1-5 & B-1-5 & B-1-5 & B-1-5 & B-1-5 \\
\hline Mineral & amphibole & amphibole & amphibole & amphibole & amphibole & amphibole & amphibole & amphibole & amphibole & amphibole & amphibole & amphibole \\
\hline Grain & 1 & 1 & 2 & 2 & 2 & 3 & 3 & 3 & 3 & 3 & 3 & 3 \\
\hline Position & core & core & rim & rim & core & rim & rim & core & rim & rim & rim & rim \\
\hline $\mathrm{SiO}_{2}$ & 42.23 & 43.36 & 42.70 & 42.58 & 42.87 & 42.25 & 42.83 & 42.66 & 42.81 & 43.14 & 43.26 & 43.16 \\
\hline $\mathrm{TiO}_{2}$ & 1.54 & 1.37 & 1.64 & 1.60 & 1.71 & 1.19 & 1.11 & 1.19 & 1.14 & 1.19 & 1.21 & 1.54 \\
\hline $\mathrm{Al}_{2} \mathrm{O}_{3}$ & 6.65 & 5.71 & 7.07 & 7.11 & 6.82 & 7.05 & 6.75 & 6.90 & 6.84 & 6.91 & 7.19 & 6.54 \\
\hline $\mathrm{Cr}_{2} \mathrm{O}_{3}$ & 0.00 & 0.00 & 0.00 & 0.00 & 0.00 & 0.00 & 0.00 & 0.02 & 0.00 & 0.02 & 0.01 & 0.00 \\
\hline $\mathrm{FeO}$ & 29.67 & 30.06 & 28.70 & 28.86 & 27.74 & 29.58 & 29.15 & 29.37 & 29.66 & 29.25 & 29.32 & 29.06 \\
\hline $\mathrm{MnO}$ & 0.72 & 0.61 & 0.64 & 0.67 & 0.63 & 0.72 & 0.72 & 0.72 & 0.74 & 0.72 & 0.66 & 0.73 \\
\hline $\mathrm{NiO}$ & 0.00 & 0.01 & 0.02 & 0.01 & 0.00 & 0.00 & 0.01 & 0.00 & 0.01 & 0.02 & 0.00 & 0.04 \\
\hline $\mathrm{MgO}$ & 3.16 & 2.98 & 3.59 & 3.56 & 4.21 & 3.25 & 3.50 & 3.52 & 3.24 & 3.25 & 3.17 & 3.28 \\
\hline $\mathrm{CaO}$ & 10.08 & 8.42 & 10.07 & 10.01 & 9.95 & 10.04 & 10.11 & 10.08 & 9.96 & 9.99 & 10.17 & 9.96 \\
\hline $\mathrm{Na}_{2} \mathrm{O}$ & 2.46 & 3.23 & 2.53 & 2.51 & 2.44 & 2.29 & 2.20 & 2.42 & 2.39 & 2.35 & 1.97 & 2.43 \\
\hline $\mathrm{K}_{2} \mathrm{O}$ & 0.95 & 0.77 & 1.03 & 0.99 & 0.97 & 1.07 & 1.07 & 1.07 & 1.11 & 1.06 & 0.98 & 0.91 \\
\hline$\Sigma$ & 97.93 & 96.86 & 98.00 & 97.91 & 97.34 & 97.45 & 97.44 & 97.95 & 97.91 & 97.91 & 97.93 & 97.65 \\
\hline $\mathrm{Si}$ & 6.76 & 6.98 & 6.76 & 6.75 & 6.79 & 6.76 & 6.83 & 6.78 & 6.81 & 6.84 & 6.84 & 6.85 \\
\hline $\mathrm{Al}(\mathrm{IV})$ & 1.23 & 1.02 & 1.24 & 1.25 & 1.21 & 1.24 & 1.17 & 1.22 & 1.19 & 1.16 & 1.16 & 1.15 \\
\hline$\Sigma \mathrm{T}$ & 7.99 & 8.00 & 8.00 & 8.00 & 8.00 & 8.00 & 8.00 & 8.00 & 8.00 & 8.00 & 8.00 & 8.00 \\
\hline $\mathrm{Al}(\mathrm{VI})$ & 0.01 & 0.06 & 0.07 & 0.08 & 0.06 & 0.08 & 0.10 & 0.07 & 0.09 & 0.13 & 0.18 & 0.08 \\
\hline $\mathrm{Ti}$ & 0.19 & 0.17 & 0.20 & 0.19 & 0.20 & 0.14 & 0.13 & 0.14 & 0.14 & 0.14 & 0.14 & 0.18 \\
\hline $\mathrm{Fe} 2$ & 3.57 & 3.64 & 3.42 & 3.44 & 3.31 & 3.56 & 3.50 & 3.51 & 3.55 & 3.49 & 3.49 & 3.47 \\
\hline $\mathrm{Fe} 3$ & 0.40 & 0.40 & 0.38 & 0.38 & 0.37 & 0.40 & 0.39 & 0.39 & 0.39 & 0.39 & 0.39 & 0.39 \\
\hline $\mathrm{Mn}$ & 0.10 & 0.08 & 0.09 & 0.09 & 0.08 & 0.10 & 0.10 & 0.10 & 0.10 & 0.10 & 0.09 & 0.10 \\
\hline $\mathrm{Mg}$ & 0.75 & 0.72 & 0.85 & 0.84 & 0.99 & 0.77 & 0.83 & 0.83 & 0.77 & 0.77 & 0.75 & 0.78 \\
\hline$\Sigma C$ & 5.02 & 5.07 & 5.00 & 5.03 & 5.02 & 5.05 & 5.05 & 5.04 & 5.04 & 5.01 & 5.03 & 5.00 \\
\hline $\mathrm{Ca}$ & 1.73 & 1.45 & 1.71 & 1.70 & 1.69 & 1.72 & 1.73 & 1.72 & 1.70 & 1.70 & 1.72 & 1.69 \\
\hline $\mathrm{Na}$ & 0.25 & 0.48 & 0.29 & 0.27 & 0.29 & 0.23 & 0.22 & 0.24 & 0.26 & 0.29 & 0.25 & 0.31 \\
\hline$\Sigma \mathrm{B}$ & 2.00 & 2.00 & 2.00 & 2.00 & 2.00 & 2.00 & 2.00 & 2.00 & 2.00 & 2.00 & 2.00 & 2.00 \\
\hline $\mathrm{Na}$ & 0.51 & 0.53 & 0.49 & 0.50 & 0.46 & 0.48 & 0.46 & 0.51 & 0.48 & 0.43 & 0.35 & 0.44 \\
\hline K & 0.19 & 0.16 & 0.21 & 0.20 & 0.20 & 0.22 & 0.22 & 0.22 & 0.23 & 0.21 & 0.20 & 0.18 \\
\hline$\Sigma \mathrm{A}$ & 0.71 & 0.69 & 0.69 & 0.70 & 0.66 & 0.70 & 0.68 & 0.72 & 0.70 & 0.65 & 0.55 & 0.62 \\
\hline
\end{tabular}

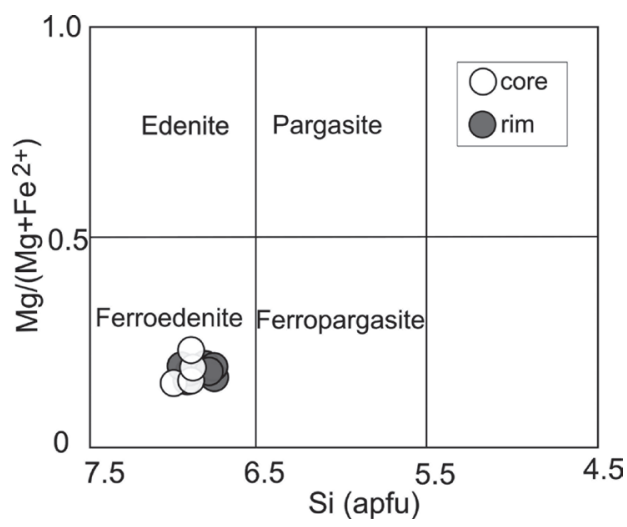

Figure 5. Classification diagram for the amphiboles from the alkali feldspar granite (B-1-5 sample) after LEAKE et al. (1997). to 0.71 , and $\mathrm{TiO}_{2}$ contents ranging from 1.57 to $3.77 \mathrm{wt} \%$. In a $\mathrm{Mg}$ vs. $\mathrm{Al}_{\text {tot }}$ diagram (Fig. 6), most biotite analyses display chemical characteristics of biotite from alkaline-peralkaline rocks (NACHIT et al., 1985).

The results of the selected EPMA analyses of feldspars from the alkali granite type 1 (B-1-5) are listed in Table 4 . The structural formula was calculated based on 8 oxygen atoms. Both K-feldspar and albite exsolution lamellae were analysed, along with single albite grains associated with clusters of mafic minerals. $\mathrm{K}$-feldspar is homogeneous with a composition $\mathrm{Or}_{94-98} \mathrm{Ab}_{2-6}$. Albite exsolution lamellae range in composition from $\mathrm{An}_{0}$ to $\mathrm{An}_{5}$, similar to albite grains, which range in composition from $\mathrm{An}_{4}$ to $\mathrm{An}_{6}$ and are homogeneous. The K-feldspar component in the plagioclase ranges from $\mathrm{Or}_{1}$ to $\mathrm{Or}_{2}$. 


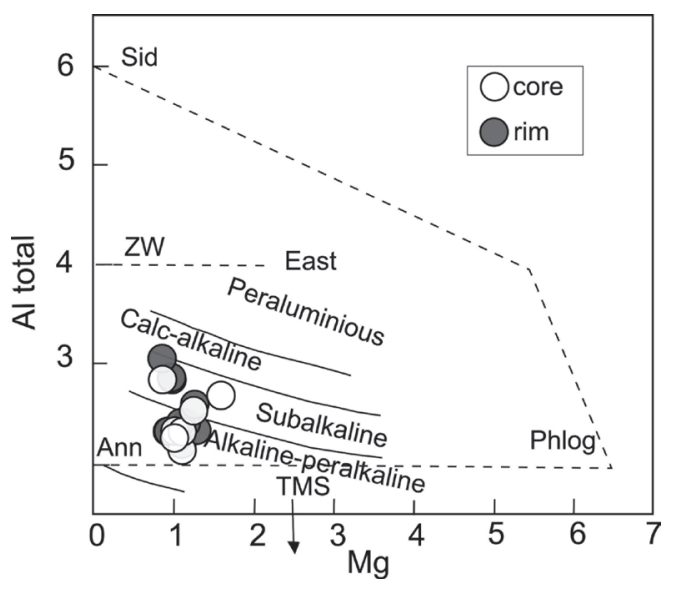

Figure 6. Discrimination diagram for biotite from the alkali feldspar granite (B1-5 sample) after NACHIT (1985). Sid; siderophyllite, Phlog: phlogopite, East: eastonite, Ann: annite, ZW: zinnwaldite.

Table 3. Selected EPMA analyses and structural formulae of biotite from the alkali granite type 1 (B-1-5). Calculated based on 24 atoms $(\mathrm{O}, \mathrm{OH})$ and 15 cations.

\begin{tabular}{|c|c|c|c|c|c|c|}
\hline Sample & B-1-5 & B-1-5 & B-1-5 & B-1-5 & B-1-5 & B-1-5 \\
\hline Mineral & biotite & biotite & biotite & biotite & biotite & biotite \\
\hline Grain & 1 & 1 & 2 & 2 & 3 & 3 \\
\hline Position & core & rim & core & rim & core & rim \\
\hline $\mathrm{SiO}_{2}$ & 29.47 & 34.46 & 36.55 & 36.39 & 36.1 & 36.32 \\
\hline $\mathrm{TiO}_{2}$ & 1.13 & 2.02 & 3.66 & 3.77 & 3.55 & 3.25 \\
\hline $\mathrm{Al}_{2} \mathrm{O}_{3}$ & 13.93 & 12.42 & 12.61 & 12.91 & 12.33 & 13.12 \\
\hline $\mathrm{Cr}_{2} \mathrm{O}_{3}$ & 0.01 & 0 & 0 & 0.03 & 0 & 0 \\
\hline $\mathrm{FeO}$ & 36.75 & 31.6 & 29.26 & 29.23 & 29.6 & 28.6 \\
\hline $\mathrm{MnO}$ & 0.31 & 0.31 & 0.27 & 0.27 & 0.7 & 0.22 \\
\hline $\mathrm{NiO}$ & 0.01 & 0.03 & 0 & 0 & 0 & 0.03 \\
\hline $\mathrm{MgO}$ & 6.48 & 5.41 & 4.47 & 4.07 & 4.8 & 4.71 \\
\hline $\mathrm{CaO}$ & 0.09 & 0.04 & 0.01 & 0.04 & 0 & 0.01 \\
\hline $\mathrm{Na}_{2} \mathrm{O}$ & 0 & 0.03 & 0.04 & 0.04 & 0.03 & 0.07 \\
\hline $\mathrm{K}_{2} \mathrm{O}$ & 1.91 & 7.25 & 8.86 & 9.18 & 9.12 & 9.07 \\
\hline$\Sigma$ & 90.29 & 93.94 & 95.75 & 95.93 & 96.21 & 95.82 \\
\hline $\mathrm{Si}$ & 4.86 & 5.47 & 5.63 & 5.6 & 5.58 & 5.61 \\
\hline $\mathrm{Al}(\mathrm{IV})$ & 2.71 & 2.32 & 2.29 & 2.34 & 2.25 & 2.39 \\
\hline$\Sigma \mathrm{T}$ & 7.57 & 7.79 & 7.92 & 7.95 & 7.83 & 7.99 \\
\hline $\mathrm{Al}(\mathrm{VI})$ & 0 & 0 & 0 & 0 & 0 & 0 \\
\hline $\mathrm{Ti}$ & 0.14 & 0.24 & 0.42 & 0.44 & 0.41 & 0.38 \\
\hline $\mathrm{Fe}^{3+}$ & 2.03 & 1.68 & 1.51 & 1.51 & 1.53 & 1.48 \\
\hline $\mathrm{Fe}^{2+}$ & 3.04 & 2.52 & 2.26 & 2.26 & 2.3 & 2.22 \\
\hline $\mathrm{Mn}$ & 0.04 & 0.04 & 0.03 & 0.03 & 0.04 & 0.03 \\
\hline $\mathrm{Mg}$ & 1.59 & 1.28 & 1.03 & 0.93 & 1.11 & 1.08 \\
\hline$\Sigma \mathrm{M}$ & 6.84 & 5.75 & 5.25 & 5.17 & 5.38 & 5.18 \\
\hline $\mathrm{Ca}$ & 0.02 & 0.01 & 0 & 0.01 & 0 & 0 \\
\hline $\mathrm{Na}$ & 0 & 0.01 & 0.01 & 0.01 & 0.01 & 0.02 \\
\hline K & 0.4 & 1.47 & 1.74 & 1.8 & 1.8 & 1.79 \\
\hline$\sum I$ & 0.42 & 1.48 & 1.75 & 1.82 & 1.81 & 1.81 \\
\hline
\end{tabular}

Table 4. Selected EPMA analyses and structural formulae of feldspar from the alkali granite type 1 (B-1-5). Calculated based on 8 oxygen atoms.

\begin{tabular}{|c|c|c|c|c|c|c|}
\hline Sample & B-1-5 & B-1-5 & B-1-5 & B-1-5 & B-1-5 & B-1-5 \\
\hline Mineral & K-feldspar & K-feldspar & albite & albite & albite & albite \\
\hline Grain & 1 & 1 & 1 & 2 & 2 & 3 \\
\hline Position & core & rim & lamella & core & rim & rim \\
\hline $\mathrm{SiO}_{2}$ & 64.24 & 64.70 & 68.06 & 67.35 & 67.60 & 67.70 \\
\hline $\mathrm{Al}_{2} \mathrm{O}_{3}$ & 17.97 & 17.93 & 19.54 & 19.81 & 19.65 & 19.68 \\
\hline $\mathrm{Fe}_{2} \mathrm{O}_{3}$ & 0.02 & 0.06 & 0.10 & 0.64 & 0.33 & 0.06 \\
\hline Bao & 0.04 & 0.02 & 0.00 & 0.02 & 0.04 & 0.01 \\
\hline $\mathrm{CaO}$ & 0.00 & 0.05 & 0.61 & 0.76 & 1.04 & 0.95 \\
\hline $\mathrm{Na}_{2} \mathrm{O}$ & 0.34 & 0.40 & 11.39 & 10.96 & 11.00 & 11.20 \\
\hline $\mathrm{K}_{2} \mathrm{O}$ & 16.58 & 15.91 & 0.18 & 0.13 & 0.08 & 0.21 \\
\hline$\Sigma$ & 99.23 & 99.11 & 99.94 & 99.67 & 99.75 & 99.87 \\
\hline $\mathrm{Si}$ & 3.00 & 3.01 & 2.98 & 2.96 & 2.97 & 2.97 \\
\hline $\mathrm{Al}$ & 0.99 & 0.98 & 1.01 & 1.03 & 1.02 & 1.02 \\
\hline $\mathrm{Fe}^{3+}$ & 0.00 & 0.00 & 0.00 & 0.02 & 0.01 & 0.00 \\
\hline $\mathrm{Ba}$ & 0.00 & 0.00 & 0.00 & 0.00 & 0.00 & 0.00 \\
\hline $\mathrm{Ca}$ & 0.00 & 0.00 & 0.03 & 0.04 & 0.05 & 0.04 \\
\hline $\mathrm{Na}$ & 0.03 & 0.04 & 0.97 & 0.93 & 0.94 & 0.95 \\
\hline K & 0.99 & 0.95 & 0.01 & 0.01 & 0.00 & 0.01 \\
\hline$\Sigma$ & 5.01 & 4.98 & 5.00 & 4.99 & 4.99 & 5.00 \\
\hline Or & 96.99 & 96.04 & 0.98 & 0.75 & 0.44 & 1.15 \\
\hline$A b$ & 3.01 & 3.70 & 96.18 & 95.61 & 94.64 & 94.44 \\
\hline An & 0.00 & 0.26 & 2.85 & 3.64 & 4.93 & 4.41 \\
\hline
\end{tabular}

\subsection{Whole-rock geochemistry}

Nine samples were analysed for major and trace elements, including REE: three samples of alkali granite type 1 (B-1-3, B-1-4, B-1-5), one sample of alkali granite type 2 (B-2-1), syenite (B-35), granite (B-6-2), rhyolite (B-6-2R) and two samples of orthogneiss (B-5-2, B-4-5). The whole-rock analyses are presented in Table 5.

The analysed rocks have a wide range of $\mathrm{SiO}_{2}$ content (61.15$77.89 \mathrm{wt} \%)$. Harker diagrams exhibit negative correlations between $\mathrm{SiO}_{2}$ and $\mathrm{Al}_{2} \mathrm{O}_{3}, \mathrm{FeO}_{\mathrm{t}}, \mathrm{CaO}, \mathrm{P}_{2} \mathrm{O}_{5}$, and $\mathrm{TiO}_{2}$ contents (Fig. 7). The $\mathrm{K}_{2} \mathrm{O}$ and $\mathrm{Na}_{2} \mathrm{O}$ contents are positively correlated with $\mathrm{SiO}_{2}$, with an exception of the highest alkali content in the syenite (B-3-5) and the lowest $\mathrm{K}_{2} \mathrm{O}$ content in the alkali granite type 2 (B-2-1). The $\mathrm{MgO}$ content is generally low (0.12-1.28 wt $\%)$, displaying a slight negative correlation with $\mathrm{SiO}_{2}$. All analysed rocks have low $\mathrm{P}_{2} \mathrm{O}_{5}$ contents $(0.01-0.19 \mathrm{wt} \%)$.

In the classification scheme of FROST et al. (2001) and FROST \& FROST (2008), based on three variables: Fe*, modified alkali-lime index (MALI) and aluminium saturation index (ASI), the analysed samples display certain differences. Regarding the $\mathrm{Fe}^{*}$, index defined as $\mathrm{FeO}_{\text {tot }} /\left(\mathrm{FeO}_{\text {tot }}+\mathrm{MgO}\right)$ (Fig. 8a), both types of alkali granite and syenite, along with one sample of the orthogneiss (B-5-2) are classified as ferroan $\left(\mathrm{Fe}^{*}=0.81-0.93\right)$, while the granite, rhyolite, and the other orthogneiss sample (B-4-5) are classified as magnesian $\left(\mathrm{Fe}^{*}=0.65-0.78\right)$. Based on the MALI index defined as $\mathrm{Na}_{2} \mathrm{O}+\mathrm{K}_{2} \mathrm{O}-\mathrm{CaO}$ (Fig. 8b), the 
Table 5. Results of whole-rock chemical analyses. $\mathrm{AG} 1$ alkali granite type $1 ; \mathrm{AG} 2$ alkali granite type $2 ; \mathrm{LOI}$ loss on ignition; $\mathrm{A} / \mathrm{NK}=\mathrm{Al} \mathrm{O}_{3} /\left(\mathrm{Na}_{2} \mathrm{O}+\mathrm{K}_{2} \mathrm{O}\right) ; \mathrm{Fe}{ }^{*}=\mathrm{FeO} \mathrm{tot}_{\text {tot }} /($ $\left.\mathrm{FeO}_{\text {tot }}+\mathrm{MgO}\right) ; \mathrm{MALI}=\mathrm{Na}_{2} \mathrm{O}+\mathrm{K}_{2} \mathrm{O}-\mathrm{CaO} ; \mathrm{ASI}=\mathrm{Al} /(\mathrm{Ca}-1.67 \times \mathrm{P}+\mathrm{Na}+\mathrm{K})$. Major element in wt\%; trace element in ppm.

\begin{tabular}{|c|c|c|c|c|c|c|c|c|c|}
\hline Sample & B-1-5 & B-1-3 & B-1-4 & B-1-5 & B-2-1 & B-5-2 & B-4-5 & B-6-2 & B-6-2R \\
\hline Rock type & syenite & AG 1 & AG 1 & AG 1 & AG 2 & orthogneiss & orthogneiss & granite & rhyolite \\
\hline $\mathrm{SiO}_{2}$ & 61.15 & 72.59 & 74.57 & 74.66 & 77.89 & 67.11 & 68.21 & 69.52 & 75.18 \\
\hline $\mathrm{TiO}_{2}$ & 0.88 & 0.27 & 0.19 & 0.2 & 0.12 & 0.62 & 0.54 & 0.41 & 0.08 \\
\hline $\mathrm{Al}_{2} \mathrm{O}_{3}$ & 17.31 & 13.71 & 12.85 & 12.84 & 12.91 & 15.18 & 15.03 & 14.54 & 14.08 \\
\hline $\mathrm{Fe}_{2} \mathrm{O}_{3}$ & 4.53 & 2.37 & 1.94 & 1.99 & 1.11 & 3.63 & 3.76 & 2.58 & 0.7 \\
\hline $\mathrm{MnO}$ & 0.07 & 0.04 & 0.03 & 0.02 & $<0.01$ & 0.09 & 0.06 & 0.06 & $<0.01$ \\
\hline $\mathrm{MgO}$ & 0.97 & 0.22 & 0.13 & 0.14 & 0.12 & 0.83 & 1.28 & 1.28 & 0.18 \\
\hline $\mathrm{CaO}$ & 2.07 & 0.84 & 0.64 & 0.55 & 0.07 & 2.32 & 1.51 & 1.05 & 0.26 \\
\hline $\mathrm{Na}_{2} \mathrm{O}$ & 4.6 & 4.5 & 4.04 & 3.92 & 5.25 & 3.24 & 3.05 & 3.7 & 3.09 \\
\hline $\mathrm{K}_{2} \mathrm{O}$ & 6.05 & 4.58 & 4.77 & 4.94 & 1.41 & 3.84 & 4.12 & 4.62 & 5.07 \\
\hline $\mathrm{P}_{2} \mathrm{O}_{5}$ & 0.19 & 0.05 & 0.03 & 0.03 & 0.01 & 0.2 & 0.18 & 0.16 & 0.03 \\
\hline LOI & 2 & 0.7 & 0.7 & 0.6 & 1 & 2.8 & 2.1 & 1.9 & 1.2 \\
\hline$\Sigma$ & 99.79 & 99.88 & 99.89 & 99.9 & 99.93 & 99.85 & 99.85 & 99.85 & 99.96 \\
\hline $\mathrm{Ba}$ & 597 & 251 & 192 & 217 & 78 & 429 & 472 & 595 & 698 \\
\hline $\mathrm{Rb}$ & 105.7 & 198.5 & 216.8 & 206.4 & 66.4 & 129.6 & 162.8 & 207.9 & 194.1 \\
\hline $\mathrm{Sr}$ & 191.2 & 48.4 & 39 & 36.5 & 100.6 & 139 & 85.4 & 171.2 & 87.4 \\
\hline$Y$ & 22.1 & 42.5 & 44.2 & 49.1 & 39.3 & 45.4 & 38.5 & 17.7 & 15.6 \\
\hline $\mathrm{Nb}$ & 35 & 43.6 & 49.2 & 43.7 & 46.4 & 16.8 & 17.2 & 16 & 11.1 \\
\hline $\mathrm{Zr}$ & 630.9 & 283.5 & 320.5 & 324.2 & 205.1 & 236.5 & 246.6 & 175.1 & 56.3 \\
\hline $\mathrm{Hf}$ & 13.8 & 8.7 & 9.9 & 10 & 8 & 5.9 & 6.8 & 4.5 & 2.4 \\
\hline Ta & 1.9 & 4.1 & 4.4 & 3.6 & 4.4 & 2.1 & 1.6 & 1.4 & 1.2 \\
\hline Th & 4.5 & 22.9 & 25.9 & 22.8 & 31 & 30.7 & 16.2 & 14.6 & 14.5 \\
\hline U & 2.1 & 4.1 & 5.5 & 5 & 4.9 & 4.8 & 8.6 & 8.2 & 4.3 \\
\hline Sc & 8 & 4 & 2 & 2 & $<1$ & 11 & 10 & 5 & 3 \\
\hline Co & 8 & 1.3 & 1.3 & 1.7 & 0.7 & 5.6 & 6.2 & 4.2 & 0.6 \\
\hline Cs & 0.9 & 4.4 & 4.2 & 4.8 & 0.9 & 11.1 & 6 & 4.6 & 3.7 \\
\hline $\mathrm{Pb}$ & 2 & 5.4 & 7.8 & 6.5 & 4 & 5 & 11.5 & 10.2 & 10.6 \\
\hline $\mathrm{Zn}$ & 30 & 54 & 50 & 40 & 11 & 57 & 33 & 43 & 6 \\
\hline $\mathrm{Ni}$ & 3.4 & 1 & 0.7 & 0.8 & 0.7 & 3.2 & 4.5 & 4.8 & 1.5 \\
\hline Ga & 23.8 & 23.2 & 22.8 & 22.5 & 23.6 & 19.5 & 22.6 & 19.2 & 14.9 \\
\hline La & 24.3 & 55.1 & 48.6 & 47.2 & 45.6 & 45.7 & 43.4 & 38.4 & 15.5 \\
\hline $\mathrm{Ce}$ & 47.3 & 105.8 & 91 & 89.6 & 86 & 90.7 & 91.9 & 70.1 & 28.3 \\
\hline $\operatorname{Pr}$ & 5.5 & 11.17 & 9.72 & 9.7 & 8.39 & 10.81 & 10.42 & 7.33 & 3.26 \\
\hline $\mathrm{Nd}$ & 21.9 & 37.8 & 35.1 & 35.6 & 29.3 & 40.6 & 39.1 & 24.8 & 11.8 \\
\hline Sm & 4.16 & 7.29 & 7.27 & 7.64 & 6.2 & 9.2 & 8.13 & 4.18 & 2.5 \\
\hline Eu & 1.33 & 0.63 & 0.52 & 0.53 & 0.24 & 1.37 & 0.96 & 0.91 & 0.52 \\
\hline Gd & 3.97 & 7.05 & 7.44 & 7.75 & 5.99 & 8.85 & 7.61 & 3.51 & 2.76 \\
\hline $\mathrm{Tb}$ & 0.66 & 1.22 & 1.21 & 1.4 & 1.11 & 1.37 & 1.21 & 0.56 & 0.42 \\
\hline Dy & 3.84 & 7.39 & 7.59 & 8.68 & 6.54 & 7.65 & 6.79 & 2.66 & 2.59 \\
\hline Ho & 0.9 & 1.51 & 1.54 & 1.74 & 1.42 & 1.64 & 1.44 & 0.59 & 0.49 \\
\hline Er & 2.46 & 4.31 & 4.64 & 5.43 & 4.4 & 4.41 & 4.01 & 1.48 & 1.5 \\
\hline $\mathrm{Tm}$ & 0.38 & 0.73 & 0.69 & 0.81 & 0.7 & 0.62 & 0.59 & 0.24 & 0.23 \\
\hline $\mathrm{Yb}$ & 2.5 & 5.03 & 4.78 & 5.33 & 4.51 & 4.47 & 4.01 & 1.77 & 1.46 \\
\hline Lu & 0.36 & 0.72 & 0.7 & 0.76 & 0.7 & 0.63 & 0.57 & 0.29 & 0.23 \\
\hline$\Sigma$ REE & 119.56 & 245.75 & 220.8 & 222.17 & 201.1 & 228.02 & 220.14 & 156.82 & 71.56 \\
\hline $\mathrm{A} / \mathrm{NK}$ & 1.23 & 1.11 & 1.09 & 1.09 & 1.27 & 1.60 & 1.59 & 1.31 & 1.33 \\
\hline $\mathrm{Fe}^{*}$ & 0.81 & 0.91 & 0.93 & 0.93 & 0.89 & 0.80 & 0.73 & 0.65 & 0.78 \\
\hline MALI & 8.58 & 8.24 & 8.17 & 8.31 & 6.59 & 4.76 & 5.66 & 7.27 & 7.9 \\
\hline ASI & 0.98 & 0.99 & 0.99 & 1.01 & 1.26 & 1.13 & 1.25 & 1.14 & 1.28 \\
\hline $\mathrm{Eu} / \mathrm{Eu}^{*}$ & 1.00 & 0.27 & 0.22 & 0.21 & 0.46 & 0.37 & 0.72 & 0.60 & 0.61 \\
\hline$(\mathrm{La} / \mathrm{Sm})_{\mathrm{cn}}$ & 3.65 & 4.72 & 4.17 & 3.86 & 4.59 & 3.10 & 3.33 & 5.74 & 3.87 \\
\hline$(\mathrm{Gd} / \mathrm{Yb})_{\mathrm{cn}}$ & 1.28 & 1.13 & 1.26 & 1.18 & 1.07 & 1.60 & 1.54 & 1.60 & 1.53 \\
\hline
\end{tabular}



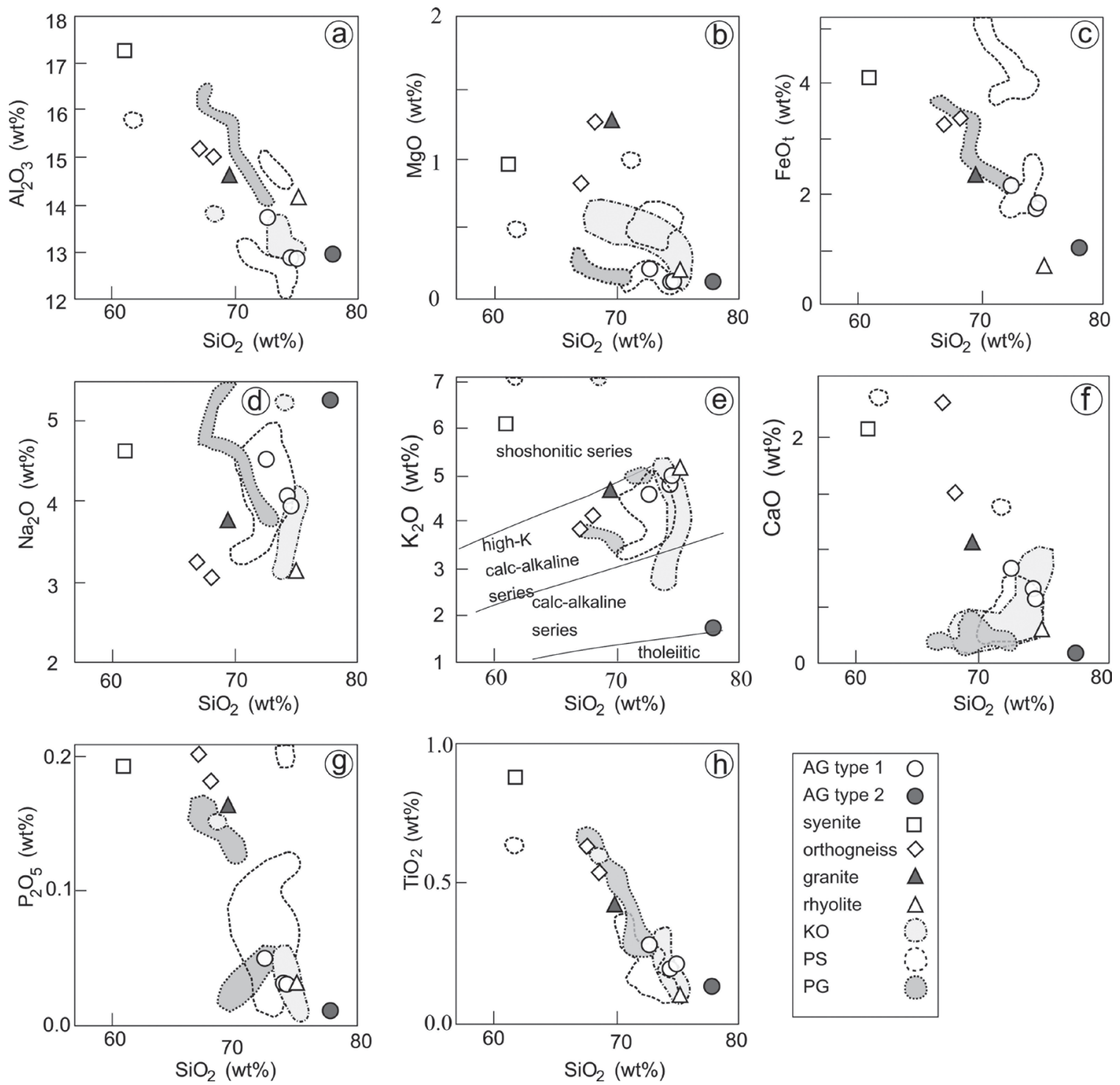

Figure 7. Harker variation diagrams of major element oxides for the analysed whole rocks. Magmatic series fields in e) after PECERILLO \& TAYLOR (1976). PG: Požeška Gora. granite (BALEN et al., 2020); KO: Kozara acidic rocks (USTASZEWSKI et al., 2009); PS Prosara granite (PAMIĆ \& INJUK, 1988).

samples mainly plot in the alkali-calcic field, except for the syenite and alkali granite type 2 , which plot in the alkalic and calcic fields, respectively. Regarding the ASI index defined as $\mathrm{Al} /(\mathrm{Ca}-1.67 \mathrm{P}+\mathrm{Na}+\mathrm{K})$, the alkali granite type 1 and the syenite are metaluminous, to slightly peraluminous while the rest of the samples are peraluminous (Fig. 8c).

Harker diagrams for selected trace elements are displayed in Fig. 9. Among the LILE (light ion lithophile elements), the Ba and $\mathrm{Sr}$ contents are negatively correlated with $\mathrm{SiO}_{2}$. Exceptions are the granite and the rhyolite in the $\mathrm{Ba}$ vs. $\mathrm{SiO}_{2}$ diagram and $\mathrm{Sr}$ vs. $\mathrm{SiO}_{2}$ diagram, as well as the alkali granite type 2 in the $\mathrm{Sr}$ vs. $\mathrm{SiO}_{2}$ diagram. $\mathrm{Rb}$ contents are positively correlated with $\mathrm{SiO}_{2}$, except for the alkali granite type 2, which is characterized by very low concentrations. Among the HFSE (high field-strength elements), $\mathrm{Zr}, \mathrm{Nb}$ and $\mathrm{Y}$ concentrations display considerable scatter, with very high $\mathrm{Zr}$ in the syenite, very low $\mathrm{Zr}$ in the rhyolite, high $\mathrm{Nb}$ in both types of alkali granite and the syenite and high $\mathrm{Y}$ in both types of alkali granite and the orthogneiss.

Primitive mantle normalized (SUN \& MCDONOUGH, 1989) trace element patterns (Fig. 10) indicate a general enrichment of incompatible over compatible elements. Both types of alkali granite and the orthogneiss display negative $\mathrm{Ba}, \mathrm{Sr}, \mathrm{P}, \mathrm{Eu}$, and $\mathrm{Ti}$ anomalies, as well as a negative $\mathrm{Nb}$ anomaly. The syenite displays distinctive positive anomalies in $\mathrm{K}$ and $\mathrm{Zr}$, as well as slightly negative anomalies in $\mathrm{Sr}, \mathrm{P}$, and $\mathrm{Ti}$. The granite and the rhyolite display negative $\mathrm{Ba}, \mathrm{Nb}, \mathrm{Sr}, \mathrm{P}$, and $\mathrm{Ti}$ anomalies and have a distinctive positive $\mathrm{Pb}$ anomaly.

Chondrite normalized (MCDONOUGH \& SUN, 1995) REE patterns are generally similar for all samples (Fig. 11), with slight LREE enrichment $(\mathrm{La} / \mathrm{Sm})_{\mathrm{cn}}=3.10-5.74$ and an almost flat HREE pattern $(\mathrm{Gd} / \mathrm{Yb})_{\mathrm{cn}}=1.07-1.60$. The main difference between the samples is a negative Eu anomaly, which is well pronounced in both types of alkali granite $\left(\mathrm{Eu} / \mathrm{Eu}^{*}=0.12-0.27\right)$, moderately pronounced in the orthogneiss $\left(\mathrm{Eu} / \mathrm{Eu}^{*}=0.37-0.46\right)$, less pronounced in the granite and rhyolite $\left(\mathrm{Eu} / \mathrm{Eu}^{*}=0.60-0.72\right)$ and absent in the syenite $\left(\mathrm{Eu} / \mathrm{Eu}^{*}=1.00\right)$.

\section{DISCUSSION}

The results of petrographic analysis indicate that the rocks presented in this study comprise a very heterogenous group. Both types of the alkali granites and the rhyolite have preserved the igneous fabric, the syenite and granite were affected by brittle 

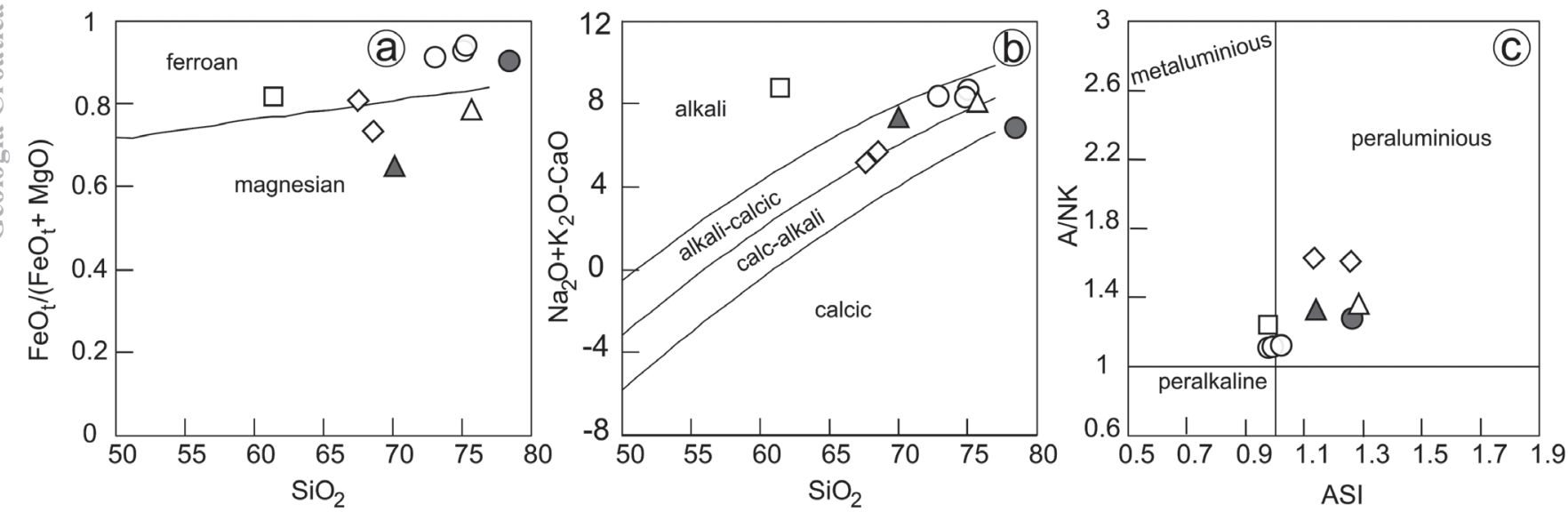

Figure 8. Classification diagrams based on the major elements after FROST et al. (2001): a) $\mathrm{SiO}_{2}-\mathrm{FeO}_{\text {tot }} /\left(\mathrm{FeO}_{\text {tot }}+\mathrm{MgO}\right) ;$ b) $\mathrm{SiO}_{2}-\mathrm{Na} \mathrm{O}_{2} \mathrm{O}+\mathrm{K}_{2} \mathrm{O}-\mathrm{CaO}$; c) $\mathrm{ASI}-\mathrm{A} / \mathrm{NK}$ Symbols as in Fig. 7.

and subordinately plastic deformation, but with the preserved igneous microstructure on a thin-section scale, while the orthogneiss was subjected to plastic deformation.

The alkali granite type 1 and syenite are characterized by their similar mineralogical composition, with the main difference of lower quartz, accompanied by higher plagioclase, biotite and hornblende modal content in the syenite compared to the alkali granite type 1 . Syenite is like the alkali granite type 1 with anhedral biotite and subhedral hornblende as ferromagnesian phases, while it differs from the alkali granite by the presence of two feldspars, defining its clear subsolvus character. The alkali granite type 1 is mainly composed of hypersolvus alkali feldspar, indicating high-temperature and low-pressure crystallization at higher crustal levels. The granophyric intergrowths imply a high degree of magma undercooling and are very common in shallow intrusions (VERNON, 2004; LOWENSTERN et al., 1997; CANDELA, 1997; DEER et al., 2013). Single albite grains, present in minor proportions $(<6 \%)$, are restricted to clusters of mafic min- erals, possibly representing an early phase of crystallization. Albite is characterized by fresh rims and cores altered to chlorite and epidote, which could be a consequence of the crystallization of these clusters at a deeper level, thus prior to final emplacement. The subhedral habit of hornblende indicates that it crystallized earlier than the interstitial biotite, which represents a late crystallizing phase. Primary hornblende indicates a minimum water content of 4 wt. $\%$ in the melt (DALL'AGNOL et al. 1999). The high Fe contents of hornblende and biotite indicate crystallization under reducing conditions.

The alkali granite type 2 is characterized by high quartz and plagioclase content as well as minor K-feldspar and accessory amounts of mafic minerals. Like the alkali granite type 1 , it is also characterized by granophyric intergrowths, but with a finer grain size.

The granite is composed of plagioclase, K-feldspar and quartz, with minor chlorite replacing a primary ferromagnesian mineral, probably biotite. The rock has porphyritic texture chara-
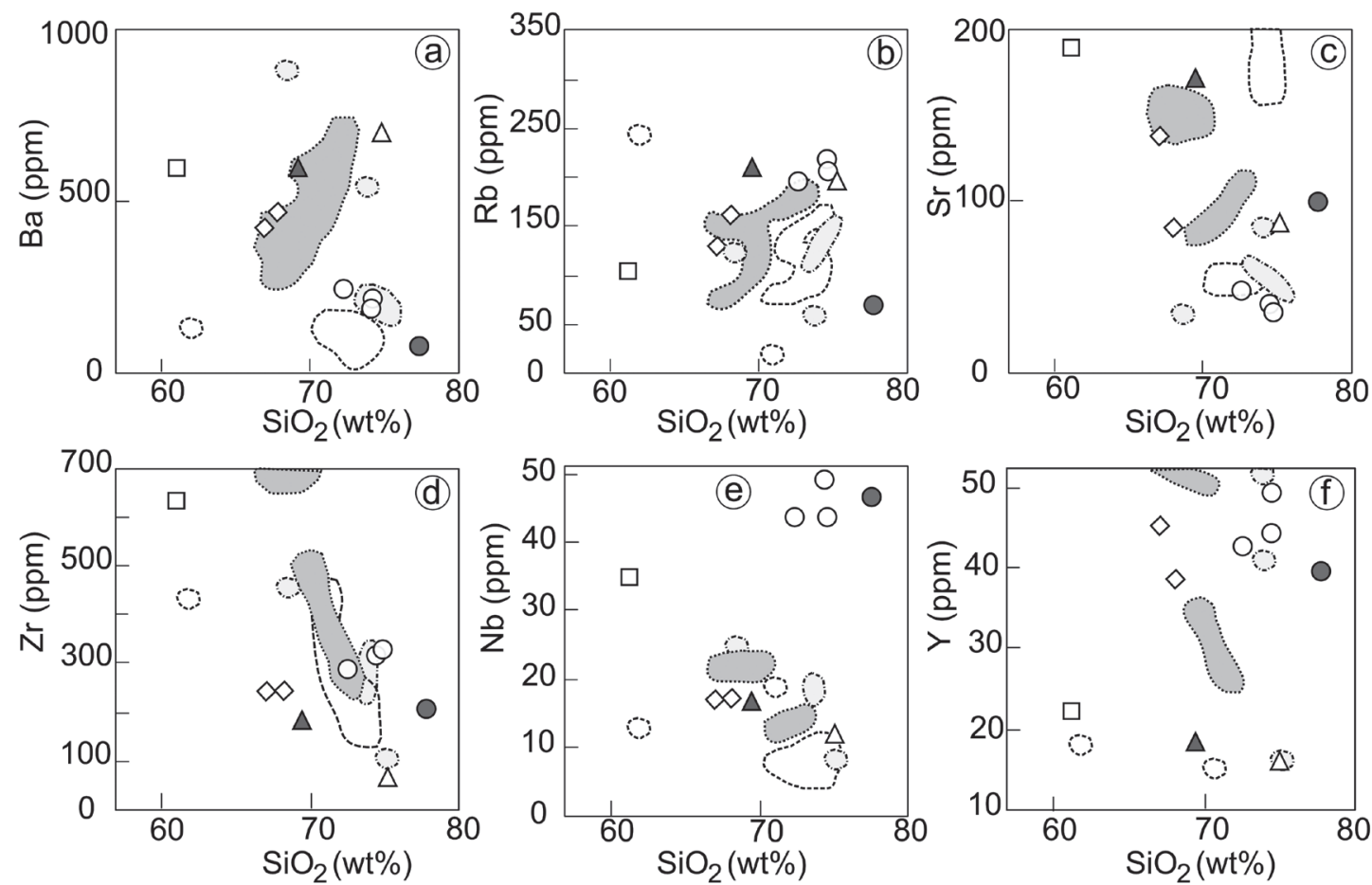

Figure 9. Harker variation diagrams of trace elements for the analysed whole rocks. Symbols as in Fig. 7. 

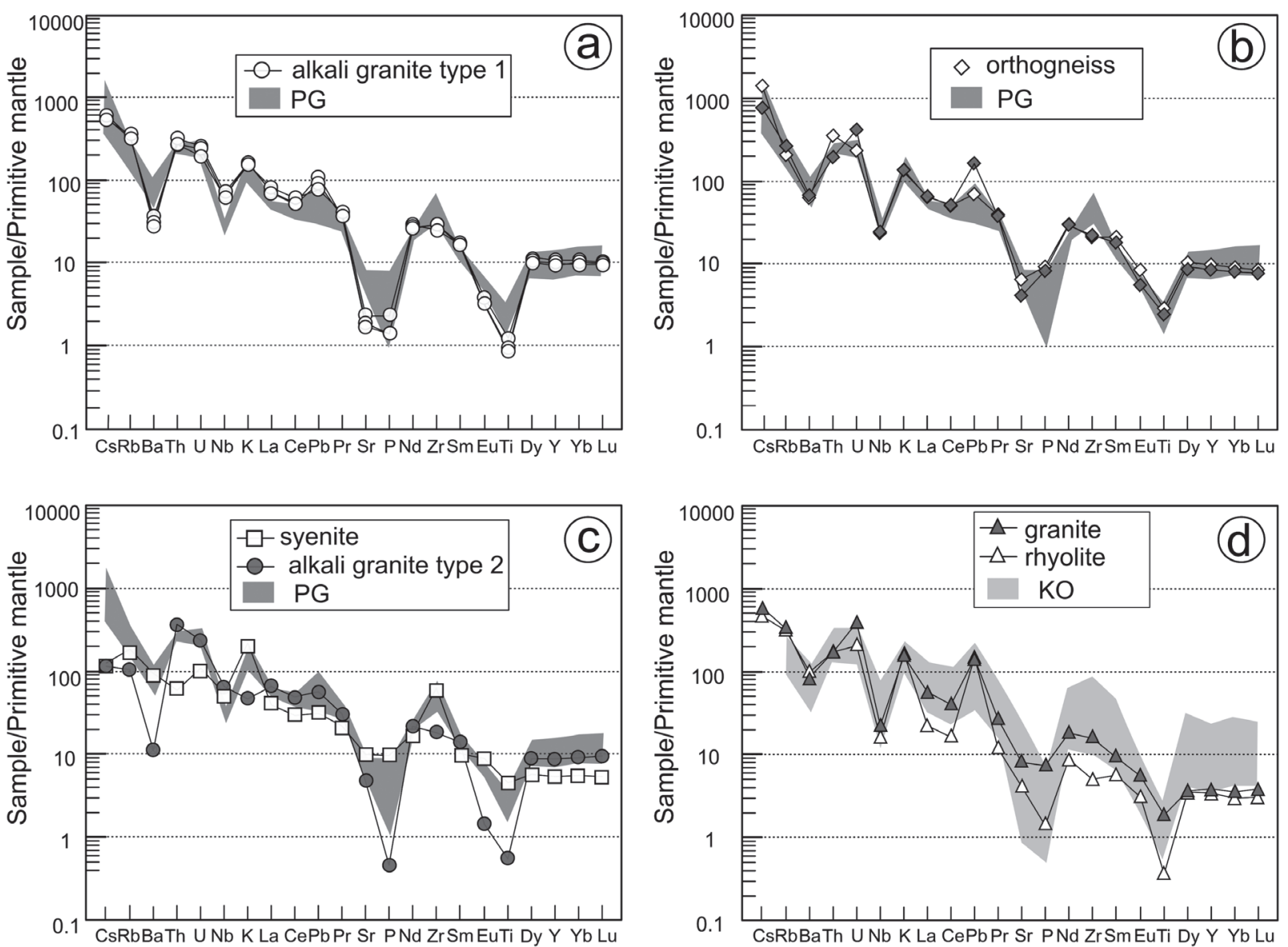

Figure 10. Primitive mantle-normalized multi-element patterns for: a) alkali granite type 1; b) orthogneiss; c) alkali granite type 2 and syenite; d) granite and rhyolite. Normalization values for the primitive mantle after SUN \& MCDONOUGH (1989). PG: Požeška Gora alkali feldspar granite (data from BALEN et al., 2020), KO: Kozara acidic rocks (USTASZEWSKI et al., 2009).
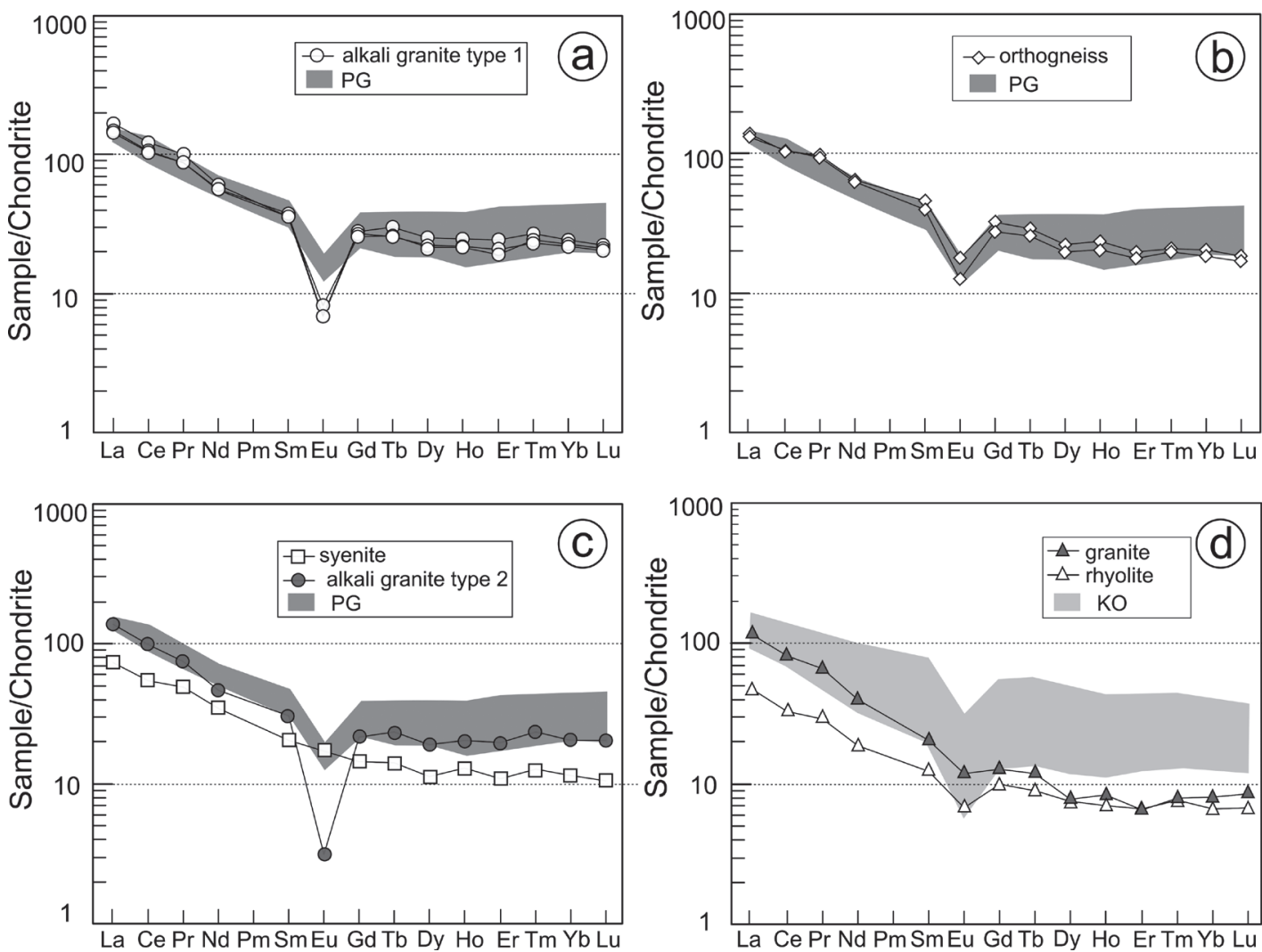

Figure 11. REE chondrite-normalized patterns for: a) alkali granite type 1 ; b) orthogneiss; c) alkali granite type 2 and syenite; d) granite and rhyolite. Normalization values for chondrite after MCDONOUGH \& SUN (1995). PG: Požeška Gora granite (data from BALEN et al., 2020), KO: Kozara acidic rocks (USTASZEWSKI et al., 2009). 
cterized by the same minerals occurring as the phenocrysts and as a part of the groundmass, which is the evidence of two-stage cooling, caused by abrupt increase in undercooling (VERNON, 2004; CANDELA, 1997). This could be a consequence of magma ascent to shallower levels or rapid loss of water, both processes pointing to crystallization in higher crustal levels. The existence of two feldspars indicates subsolvus crystallization, probably due to high water pressure (DEER et al., 2013).

The rhyolite is characterized by aphyric texture with sparse quartz and feldspar phenocrysts in the spherulitic and micropoikilitic groundmass. According to BACHMAN \& BERGANTZ (2004), the crystal-poor rhyolites are the products of interstitial melt extraction from the silicic crystal mush preserved as granitoid after the crystallization.

The orthogneiss is characterized by higher quartz and mica contents, compared to the analysed granitoids, as well as a predominance of plagioclase over K-feldspar. Quartz ribbons with elongated subgrains and undulose extinction, as well as porphyroclasts of K-feldspar with intracrystalline cracks and undulose extinction indicate plastic deformation under the low-grade metamorphic conditions.

\subsection{Geothermobarometry}

The $\mathrm{Al}$ content in amphibole can be used for estimation of the pressure (depth) of emplacement in hornblende-bearing granite (HAMMASTROM \& ZEN, 1986; HOLLISTER et al., 1987; JOHNSON \& RUTHERFORD, 1989; SCHMIDT, 1992; MUTCH et al., 2016). Based on the microstructural characteristics of the alkali granite type 1 indicating shallow emplacement, we applied the geobarometer of JOHNSON \& RUTHERFORD (1989), since it was specifically developed for low pressure conditions. Our pressure estimation for the alkali granite type 1 ranges between 1.2 and $2.5 \mathrm{kbar}$, with the higher-pressure value corresponding to a depth of $\sim 8 \mathrm{~km}$. According to ANDERSON \& SMITH (1995), the Al-in-hornblende geobarometer is suitable only for amphiboles with $\mathrm{Fe} /(\mathrm{Fe}+\mathrm{Mg})<0.65$ and granitic systems with $\mathrm{T}<800^{\circ} \mathrm{C}$, otherwise the pressure will be overestimated. The Fe/(Fe+Mg) ratio of the analysed amphibole from the alkali feldspar granite (B-1-5) is 0.79 and 0.85 , so the pressures calculated with Al-inhornblende barometer should be considered as an upper pressure limit.

The magmatic temperatures of the analysed igneous rocks were estimated by a zircon saturation thermometer (WATSON \& HARRISON, 1983). This temperature refers to an estimate of

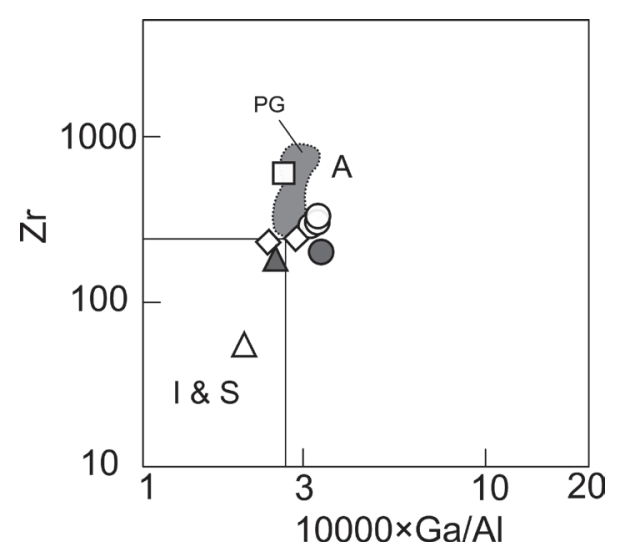

Figure 12. Discrimination diagram for granites after WHALEN et al. (1987). I \& S: I- and S-type granites, A: A-type granites. Symbols as in Fig. 7 PG: Požeška Gora granite (data from BALEN et al., 2020). melt temperature before extensive crystallization (MILLER et al., 2003). The thermometer is based on a zircon solution solubility model defined as $\ln \mathrm{Dzr}=12900 / \mathrm{T}-0.85 \times(\mathrm{M}-1)-3.80$, where Dzr is distribution coefficient of $\mathrm{Zr}$ between zircon and melt, $\mathrm{T}$ is the absolute temperature $(\mathrm{K})$ and $\mathrm{M}$ is the cationic ratio defined as $\mathrm{M}=(\mathrm{Na}+\mathrm{K}+2 \mathrm{Ca}) /(\mathrm{Al} \times \mathrm{Si})$. All samples are within the calibration range for the value of cationic ratio $\mathrm{M}$ (1.09-1.49), except for the syenite $(\mathrm{M}=1.79)$. Estimated temperatures are relatively high for the alkali granite type $1\left(831-850^{\circ} \mathrm{C}\right)$ and type $2\left(832^{\circ} \mathrm{C}\right)$, lower for granite $\left(797^{\circ} \mathrm{C}\right)$ and lowest for the rhyolite $\left(721^{\circ} \mathrm{C}\right)$. A revised zircon saturation thermometer (BOEHNKE et al., 2013) defined by $\ln \mathrm{Dzr}=(10108 \pm 32) / \mathrm{T}-(1.16 \pm 0.15)(\mathrm{M}-1)-(1.48 \pm 0.09)$ yielded lower temperatures, namely $789-813^{\circ} \mathrm{C}$ for alkali granite type 1 , $805^{\circ} \mathrm{C}$ for alkali granite type $2,754^{\circ} \mathrm{C}$ for granite and $673^{\circ} \mathrm{C}$ for rhyolite. The obtained temperatures are generally in line with microstructural observations.

\subsection{Granite typology}

The geochemical discrimination scheme of FROST et al. (2001) clearly discriminates two groups of analysed rocks, ferroan, consisting of both types of alkali granite, syenite and one sample of orthogneiss and magnesian, consisting of granite, rhyolite and the other sample of orthogneiss. According to FROST \& FROST (2011), the category of ferroan granites is equal to the A-type granite.

Alkali granite type 1 is indeed an A-type granite as defined by LOISELLE \& WONES, (1979); COLLINS et al. (1982), WHALEN et al. (1987) and EBY (1990) which is evident from its mineral chemistry (Fe-rich rich biotite and Fe-rich hornblende as ferromagnesian phases), major element contents (high $\mathrm{FeO}_{\mathrm{t}} /$ $\left(\mathrm{MgO}+\mathrm{FeO}_{\mathrm{t}}\right), \mathrm{K}_{2} \mathrm{O}, \mathrm{Na}_{2} \mathrm{O}$, accompanied by low $\mathrm{CaO}, \mathrm{MgO}$, and $\mathrm{P}_{2} \mathrm{O}_{5}$ ) i.e. ferroan, alkali-calcic and metaluminous to slightly peraluminous character, as well as trace element concentrations (high $\mathrm{REE}^{3+}, \mathrm{Rb}, \mathrm{Zr}, \mathrm{Nb}$ and $\mathrm{Y}$, and low $\mathrm{Ba}, \mathrm{Sr}$ and $\mathrm{Eu}$ ). It is characterized by a REE pattern typical for A-type granites, with a pronounced negative Eu anomaly, significant LREE enrichment and an almost flat HREE (PAPOUTSA et al., 2015).

The alkali granite type 2 also has an A-type affinity, as implied by its major element chemistry (high $\mathrm{FeO}_{\mathrm{t}} /\left(\mathrm{MgO}+\mathrm{FeO}_{\mathrm{t}}\right)$, $\mathrm{Na}_{2} \mathrm{O}$, low $\mathrm{CaO}$ and $\mathrm{MgO}$ ), trace element concentrations (high $\mathrm{REE}^{3+}, \mathrm{Nb}$ and $\mathrm{Y}$, low $\mathrm{Ba}$ and $\mathrm{Eu}$ ) as well as the shape of the REE pattern. The exception from the classical A-type granite definition is low $\mathrm{K}_{2} \mathrm{O}$ and $\mathrm{Rb}$, as well as a high $\mathrm{Sr}$ concentration. Consequently, it is a ferroan, calcic (due to low $\mathrm{K}_{2} \mathrm{O}$ ) and peraluminous rock.

The syenite, with its ferroan, alkali and metaluminous character is geochemically similar to the alkali granite type 1 in terms of major and trace element contents and could also be defined as an A-type rock. The main differences are positive $\mathrm{K}$ and $\mathrm{Zr}$ anomalies in a primitive mantle normalized trace element plot and a smooth chondrite normalized REE pattern with no Eu anomaly in the syenite. The high $\mathrm{Ga} / \mathrm{Al}$ of both types of alkali granite and the syenite confirms the A-type affinity of these rocks (WHALEN et al., 1987, Fig. 12). Additionally, the A-type affinity of both types of alkali granite is corroborated by within-plate character based on $\mathrm{Rb}, \mathrm{Nb}$, Ta, Y and $\mathrm{Yb}$ concentrations, while the syenite, due to lower Ta is not exclusively placed in the within-plate field (PEARCE et al., 1984, Fig. 13).

On the other hand, the granite differs from the above described A-type rocks by its magnesian character, lower $\mathrm{Zr}, \mathrm{Nb}$ and $\mathrm{Y}$ concentrations, less pronounced Eu negative anomaly, higher $\mathrm{Ba}, \mathrm{Sr}$ and LREE/HREE. The granite probably corre- 

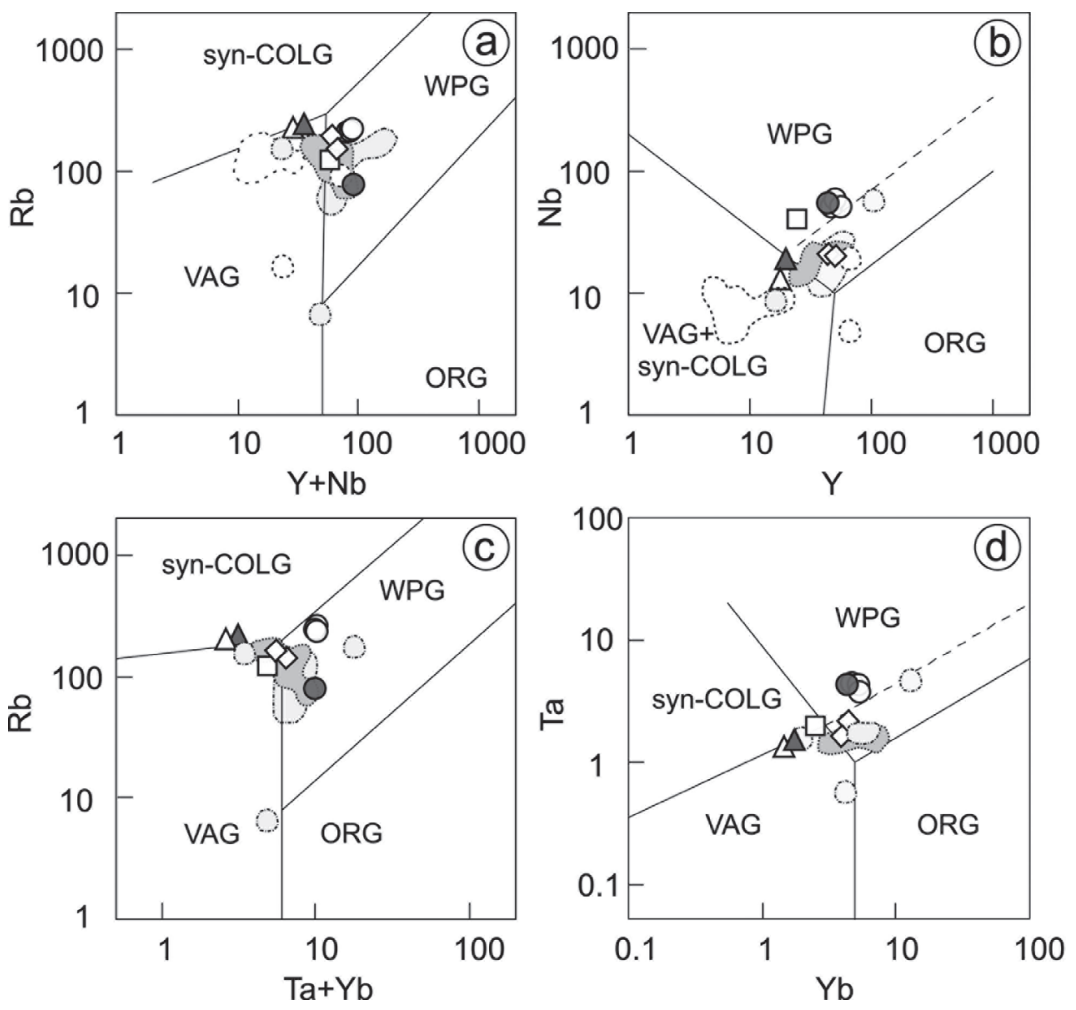

Figure 13. Discrimination diagrams for granites after PEARCE et al. (1984). WPG: within plate granite, syn-COLG: syncollisional granite; VAG: volcanic arc granite; ORG: ocean ridge granite. Symbols as in Fig. 7

sponds to an S-type rock since it is peraluminous, characterized by low $\mathrm{CaO}$ at given $\mathrm{FeO}_{\mathrm{t}}$, as well as relatively high $\mathrm{Pb}, \mathrm{Rb}$ and $\mathrm{K}_{2} \mathrm{O}$ (CHAPELL \& WHITE, 1974, 2001). On the other hand, resolving the typology of this rock is not straightforward because it is also characterized by relatively high $\mathrm{Na}_{2} \mathrm{O}$ and $\mathrm{SiO}_{2}<72$ wt.\%. The rhyolite is magnesian, alkali-calcic and peraluminous like granite, with similar trace element and REE patterns.

The orthogneiss generally has primitive mantle normalized trace element patterns and chondrite normalized REE patterns, very similar to the alkali granite type 1 . Y concentration is high and similar to both types of alkali granite. It plots on the border of an A-type and I/S type fields in the $\mathrm{Zr}$ vs. $10000^{*} \mathrm{Ga} / \mathrm{Al}$ diagram (Fig. 12), and mainly displays within-plate character based on $\mathrm{Rb}, \mathrm{Nb}, \mathrm{Ta}, \mathrm{Y}$ and $\mathrm{Yb}$ concentration (Fig. 13). On the other hand, it is characterized by moderate $\mathrm{FeO}_{\mathrm{t}} /\left(\mathrm{MgO}+\mathrm{FeO}_{\mathrm{t}}\right)$, higher $\mathrm{CaO}$ and $\mathrm{P}_{2} \mathrm{O}_{5}$, as well as lower $\mathrm{Nb}$, unlike the alkali granite types 1 and 2. According to BONIN (2008), the Y content and REE patterns are reliable indicators of A-type affinity of orthogneiss, while major element contents and $\mathrm{Nb}$ concentrations are more prone to change during the metamorphic processes. Thus, it is possible that a protolith of porphyritic granite is similar to the analysed alkali granite type 1 .

\subsection{Petrogenesis}

The similarity between alkali granite type 1 and syenite has already been mentioned in the previous section. The positive $\mathrm{K}$ and $\mathrm{Zr}$ anomalies, an $\mathrm{Eu} / \mathrm{Eu}^{*} \sim 1$ of the syenite and enrichment in $\mathrm{Ba}$ compared to alkali granite type 1, point to K-feldspar, plagioclase and zircon crystal accumulation and a silicic cumulate origin for this rock (SCHAEN et al., 2017). Significant negative Ba, Sr and Eu anomalies in the alkali granite type 1, along with the fact that these anomalies are absent ( $\mathrm{Ba}$ and $\mathrm{Eu}$ ) or considerably less pronounced $(\mathrm{Sr})$ in the syenite, indicate that these rocks could be re- lated through the processes of fractional crystallisation/crystal accumulation. The process of feldspar accumulation also explains the elevated MALI index and resulting offset to the alkali series for the syenite (FROST \& FROST, 2008). The less pronounced Ti negative anomaly followed by the absence of $\mathrm{Nb}$ depletion in syenite and higher $\mathrm{Nb} / \mathrm{Ta}$ in syenite (18.42) compared to alkali granite type $1(10.63-12.13)$ points to biotite accumulation (WERE \& KEPPLER, 2021).

The alkali granite type 2 , with its REE pattern similar to alkali granite type 1 and more pronounced Eu depletion could represent the more fractionated member of the same series, but lower $\mathrm{K}_{2} \mathrm{O}$ and $\mathrm{Rb}$, accompanied by higher $\mathrm{Na}_{2} \mathrm{O}$ and $\mathrm{Sr}$ cannot be explained by a fractionation process. While alteration of the alkali granite type 2 could be a possible cause of such differences, a different parental magma or alternative evolutionary process(es) cannot be excluded. FROST \& FROST (2011) claim that ferroan calcic peraluminous granites are theoretically possible, but they have not identified any examples of this peculiar composition.

Since the REE of the alkali granite type 1 and the syenite, display almost parallel chondrite normalized patterns, apart from the $\mathrm{Eu} / \mathrm{Eu}^{*}$, it is reasonable to conclude that this pattern was not affected by the late-stage process of fractional crystallization/ crystal accumulation and can be used as a proxy for the source rock or parental magma composition. The enrichment in LREE, coupled with the flat HREE pattern and negative $\mathrm{Sr}$ anomaly, common to both syenite and alkali feldspar granite type 1 indicate the presence of clinopyroxene/hornblende and plagioclase, along with the absence of garnet in the residual or early-stage fractionating assemblage (HANSON, 1979). The negative $P$ and $\mathrm{Ti}$ anomalies could be a result of apatite and Ti-bearing mineral in the residual or early-stage fractionating assemblage (e.g. ROLLINSON \& PEASE, 2021). 
The origin of A-type granites, particularly the nature of their magma source, is a matter of long-lasting debate (summarized in BONIN, 2007). Proposed models for A-type magma generation include: a) direct fractionation of mantle-derived magma that could be accompanied by the interaction with crustal rocks (EBY, 1990; CHEN et al. 2009); b) partial melting of crustal material for instance, previously melted lower crustal granulitic residues (COLLINS et al., 1982; WHALEN et al., 1987), or tonalitic to granodioritic rocks that suffered no previous episode of partial melting (CREASER et al., 1991). According to FROST \& FROST (2011), ferroan granites, their substitute for A-type granite term, could be generated by extreme differentiation of basalts, by partial melting of tonalitic to granodioritic crustal rocks, or by a combination of these processes.

EBY (1992) distinguished two types of A-type granite: $A_{1}$-type granite, derived from an ocean-island basalt (OIB)-like source and possibly related to intracontinental rifting events and an $\mathrm{A}_{2}$-type granite, derived from a crustal source and related to post-collisional extension. Since the author explicitly claims that his discrimination can be applied only on rocks that plot in the A-type granite field of WHALEN et al. (1987) and the within-plate granite field of PEARCE et al. (1984), we plotted exclusively the alkali granite type 1 and 2 in the discrimination diagrams $\mathrm{Y}-\mathrm{Nb}-\mathrm{Ce}$ and Y-Nb-3Ga (Fig. 14). Based on these diagrams, both alkali granite types 1 and 2 plot in the $A_{1}$-type field. This should be taken with caution due to a restricted dataset and the fact that they plot close to the boundary with the $\mathrm{A}_{2}$-field.

On the other hand, the granite probably represents an S-type granite, originating from the melting of immature, feldspar-rich metasedimentary crustal rock. Rhyolite could originate from the melting of similar parental material, but the dataset is insufficient to establish any reliable connection between these rocks. Weak Eu negative anomalies in the granite and the rhyolite, accompanied by negative $\mathrm{Sr}$ and $\mathrm{Ba}$ anomalies point to residual or fractionated plagioclase and K-feldspar, while HREE depletion in the porphyritic granite suggests residual/fractionated garnet. Negative anomalies of $\mathrm{Nb}$ and $\mathrm{Ti}$ point to a residual/fractionated Ti-phase, while a negative $\mathrm{P}$ anomaly indicates residual/fractionated apatite.

\subsection{Possible surface counterparts}

PAMIĆ \& LANPHERE (1991) considered the granites and gneiss from the studied boreholes as the Hercynian S-type granites and migmatites of the Papuk Mts. However, according to PAMIĆ et al. (1996) the S-type granites of Papuk have a lower alkali content $\left(\mathrm{K}_{2} \mathrm{O}+\mathrm{Na}_{2} \mathrm{O}\right)$, lower $\mathrm{K}_{2} \mathrm{O} / \mathrm{Na}_{2} \mathrm{O}$ ratio, higher $\mathrm{CaO}$, along with different trace element concentrations (higher $\mathrm{Ba}$ and $\mathrm{Sr}$, lower $\mathrm{Rb}$, etc.), so granites and gneiss from this study cannot be correlated with the Papuk S-type granites.

The only known A-type granites in the wider region of eastern Croatia, northern Bosnia and Herzegovina or north-western Serbia are the A-type granites associated with Late Cretaceous bimodal magmatism of the Sava Zone on the Požeška Gora (PAMIĆ, 1987; BALEN et al., 2020) and the Prosara. (PAMIĆ \& INJUK, 1988; USTASZEWSKI et al., 2010). The A-type granite of the Požeška Gora is a hypersolvus granite, as is the alkali granite type 1 from this study and displays many similarities with both types of alkali granite and syenite from this study, including trace element and REE patterns, high $\mathrm{FeO}_{\mathrm{t}} /\left(\mathrm{MgO}+\mathrm{FeO}_{\mathrm{t}}\right), \mathrm{K}_{2} \mathrm{O}$ and $\mathrm{Na}_{2} \mathrm{O}$, accompanied by low $\mathrm{CaO}$ and $\mathrm{MgO}$ (Fig.7). On the other hand, the A-type granite of the Požeška Gora is an $\mathrm{A}_{2}$-type according to EBY (1992) and it is characterized by lower $\mathrm{Nb}$ (Fig. 9). The Prosara A-type granite, which was subjected to lowgrade solid-state deformation (USTASZEWSKI et al., 2010), also displays similar major element and trace element geochemistry (PAMIĆ \& INJUK, 1988; Fig. 7, Fig. 9). However, the Nb concentration for the Prosara A-type granite is even lower than the Požeška Gora A-type granite (Fig. 13). If the studied A-type granites are indeed Late Cretaceous and if they represent a protolith for the studied orthogneiss, then the low-grade metamorphic event that produced this gneiss could be the same as that proposed for the Prosara A-type granite, that is a Palaeogene compressional phase (USTASZEWSKI e al., 2010).

Rhyolites are an important part of the Late Cretaceous bimodal complexes of the Požeška Gora (PAMIĆ et al., 2000) and the Kozara (USTASZEWSKI et al., 2009). The rhyolite from this study compared to the Požeška Gora rhyolite and the Kozara rhyolite has a different REE signature (Fig.11) with higher Eu/Eu*, $(\mathrm{Gd} / \mathrm{Yb})_{\mathrm{cn}}$ and $(\mathrm{La} / \mathrm{Sm})_{\mathrm{cn}}$. The granite is significantly different from the A-type granites of the Sava Zone as well as the A-type rocks analysed in this study.

However, it is impossible to set a reliable correlation based on geochemical and petrographic data, so any connection with surface outcrops remains open and unconstrained. Further work focused on radiometric and isotope data is necessary for resolving a number of open questions presented in this paper. Radiometric age data would, besides putting the analysed rocks in a
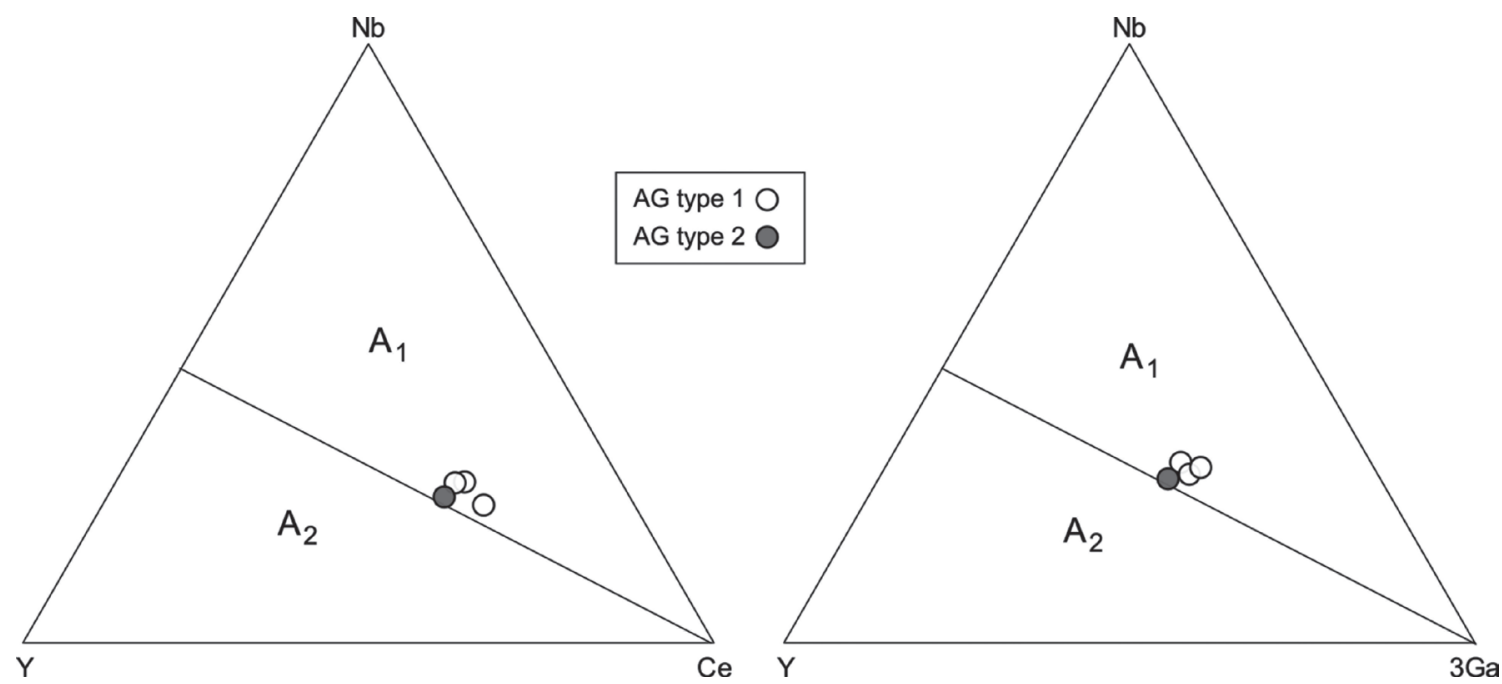

Figure 14. Discrimination diagrams for A-type granites after EBY (1992). 
regional geological context, aid in testing the hypothesis of the presumed co-genetic relationship of the alkali granite type 1 and the syenite, or speculation about the alkali granite type 1-like protolith for the analysed orthogneiss. Additionally, isotope data are necessary for resolving the origin of the parental magmas, especially for the analysed alkali granites, which were according to the classification of EBY (1992) derived from an enriched mantle source. Since the A-type granites are indicators of large-scale extensional events (e.g. BONIN, 2007), the rocks presented in this study could be crucial for understanding the tectonic evolution of the study area and consequently the wider area of the Europe-Adria suture zone.

\section{CONCLUSIONS}

This paper presented analysis of the petrography and geochemistry of the granitoids and related rocks from the pre-Neogene basement of the SSD. These data enabled the recognition of two types of alkali granite, granite and syenite. The spatially related orthogneiss and rhyolite were also included in this study. Although the dataset is relatively restricted, several conclusions can be drawn.

The alkali granite type 1 is a hypersolvus granite, crystallized from a high-temperature shallow intrusion (with $<8 \mathrm{~km}$ depth of intrusion). The overall characteristics of this rock point to an A-type affinity, while the trace element ratios $(\mathrm{Y} / \mathrm{Nb})$ indicate the mantle source of the parental magma (i.e. $A_{1}$-type affinity). The syenite displays geochemical and mineralogical features like the alkali granite type 1, but with the characteristics of a silicic cumulate rock. The alkali granite type 2 is an A-type granite too, with $\mathrm{A}_{1}$-type affinity, but compared to the alkali granite type 1 , is characterized by low $\mathrm{K}_{2} \mathrm{O}$ and $\mathrm{Rb}$, accompanied by high $\mathrm{Na}_{2} \mathrm{O}$, which could be a consequence of alteration or different parental magma/evolutionary process(es). These rocks could tentatively be related to the A-type granites of the Sava Zone.

The granite is porphyritic, indicating a shallow-intrusion origin, but with a subsolvus two-feldspar assemblage, pointing to crystallization under higher water pressure compared to the alkali granites presented. Overall geochemical characteristics, namely the magnesian character, clearly suggest that it is not genetically related to the A-type rocks described above. Geochemical characteristics suggest the S-type affiliation of this rock. The associated rhyolite displays certain similarities with the granite but setting any connection between these rocks is impossible based on the presented dataset.

\section{ACKNOWLEDGEMENT}

The authors are grateful to Mario MATOŠEVIĆ and Lutz NASDALA for their critical comments that helped to improve an early version of the manuscript. Neven ŠUICA is acknowledged for graphical help. We are very greatful to three anonymus reviewers for valuable reviews. This work was supported by the Croatian Ministry of Science, Education and Sports (195-1951293-0237).

\section{REFERENCES}

ANDERSON, J.L. \& SMITH, D.R. (1995): The effects of temperature and $\mathrm{fO}_{2}$ on the Alin-hornblende barometer- Am. Mineral., 80, 549-559.

BACHMAN, O. \& BERGANTZ, G.W. (2004): On the Origin of Crystal-poor Rhyolites: Extracted from Batholitic Crystal Mushes.- J Petrology, 45/8, 1565-1582. doi: 10.1093/petrology/egh019

BALEN, D., SCHNEIDER, P., MASSONNE, H.-J., OPITZ, J., LUPTÁKOVÁ, J., PUTIŠ, M. \& PETRINEC, Z. (2020): The Late Cretaceous A-type alkali-feldspar granite from Mt. Požeška Gora (N Croatia): Potential marker of fast magma ascent in the Europe -Adria suture zone.- Geol. Carpathica, 71, 361-381. doi: 10.31577/GeolCarp.71.4.5
BALEN, D., MASSONNE, H.-J. \& PETRINEC, Z. (2015): Collision-related Early Paleozoic evolution of a crustal fragment from the northern Gondwana margin (Slavonian Mountains, Tisia Mega-Unit, Croatia): Reconstruction of the P-T path, timing and paleotectonic implications.- Lithos, 232, 211-228. doi: 10.1016/j.lithos.2015.07.003

BALEN, D., HORVATH, P., TOMLJENOVIĆ, B., FINGER, F., HUMER, B., PAMIĆ, J. \& ARKAI, P. (2006): A record of pre-Variscan Barrovian regional metamorphism in the eastern part of the Slavonian Mountains (NE Croatia).- Mineral. Petrol., 87, 143-162. doi: 10.1007/s00710-006-0120-1

BELAK, M., HALAMIĆ, J., MARCHIG, V. \& TIBLJAŠ, D. (1998): Upper CretaceousPalaeogene Tholeiitic Basalts of the Southern Margin of the Pannonian Basin: Požeška gora Mt (Croatia).- Geol. Croat., 51, 163-174.

BOEHNKE P., WATSON E.B., TRAIL D., HARRISON T.M. \& SCHMITT A.K. (2013): Zircon saturation re-revisited.- Chemical Geology, 351, 324-334. doi: 10.1016/j. chemgeo.2013.05.028

BONIN, B. (2008): Death of super-continents and birth of oceans heralded by discrete Atype granite igneous events: the case of the Variscan-Alpine Europe-- J. Geosci., 53, 237-252. doi: 10.3190/jgeosci.036

BONIN (2007): A-type granites and related rocks: Evolution of a concept, problems and prospects.- Lithos, 97, 1-29. doi: 10.1016/j.lithos.2006.12.007

BRKIĆ, M., GALOVIĆ, I. \& BUZALJKO, R. (1989): Osnovna geološka karta SFRJ 1:100 000 list Vinkovci [Basic Geological Map of SFRY 1:100 000, Sheet Vinkovci - in Croatian].- Savezni geološki zavod, Beograd.

CANDELA, P.A. (1997): A Review of Shallow, Ore-related Granites: Textures, Volatiles, and Ore Metals.- J Petrology, 38/12, 1619-1633. doi: 10.1093/petroj/38.12.1619

CHAPPELL B.W. \& WHITE A.J.R. (2001): Two contrasting granite types: 25 years later.Australian Journal of Earth Sciences, 48, 489-499. doi: 10.1046/j.1440-0952.2001.00882.x

CHAPPELL, B.W. \& WHITE, A.J.R. (1974): Two contrasting granite types.- Pacific Geology $8,173-174$.

CHEN, L., MA, C.Q., SHE, Z.B., MASON, R., ZHANG, J.Y. \& ZHANG, C. (2009): Petrogenesis and tectonic implications of A-type granites in the Dabie orogenic belt, China: geochronological and geochemical constraints.- Geol Mag, 146, 638-651. doi: $10.1017 / \mathrm{S} 0016756808005918$

COLLINS, W.J., BEAMS, S.D., WHITE, A.J.R. \& CHAPPELL, B.W. (1982): Nature and Origin of A-type Granites with Particular Reference to Southeastern Australia.-Contrib. to Mineral. Petrol., 80, 189--200. doi: 10.1007/BF00374895

CREASER, R.A., PRICE, R.C. \& WORMALD, R.J. (1991): A-type granites revisited: Assessment of a residual-source model.- Geology, 19, 63-166. doi: 10.1130/0091-7613(1991)019\%3C0163:ATGRAO\%3E2.3.CO;2

CVETKOVIĆ, V., ŠARIĆ, K., GRUBIĆ, A., CVIJIĆ, R. \& MILOŠEVIĆ, A. (2014): The Upper Cretaceous ophiolite of North Kozara - remnants of an anomalous mid-ocean ridge segment of the Neotethys?- Geol. Carpathica, 65, 117-130. doi: 10.2478/geoca-2014-0008

ČIČULIĆ-TRIFUNOVIĆ, M. \& GALOVIĆ, I. (1984): Osnovna geološka karta SFRJ 1:100 000: List Bačka Palanka [Basic Geological Map of SFRY 1:100 000, Sheet Bačka Palanka].- Savezni geološki zavod, Beograd.

DALL'AGNOL, R., SCAILLET, B. \& PICHAVANT, M. (1999): An Experimental Study of a Lower Proterozoic A-type Granite from the Eastern Amazonian Craton, Brazil.J. Petrol. 40, 11, 1673-1698. doi: 10.1093/petroj/40.11.1673

DEER, W.A., HOWIE, R.A. \& ZUSSMAN, J. (2013): An Introduction to the Rock-Forming Minerals.- Mineralogical Society, London, 498 p. doi: 10.1180/DHZ

DE LA ROCHE, H., LETERRIER, J., GRANDCLAUDE, P. \& MARCHAL, M. (1980): A classification of volcanic and plutonic rocks using R1R2-diagram and major element analyses - its relationships with current nomenclature.- Chem. Geol. 29, 183210.

EBY, G.N. (1992): Chemical subdivision of the A-type granitoids: Petrogenetic and tectonic implications.- Geology, 20, 641-644.

EBY, G.N. (1990): The A-type granitoids: A review of their occurrence and chemical characteristics and speculations on their petrogenesis.- Lithos, 26, 115-134. doi: 10.1016/0024-4937(90)90043-Z

FROST, B.R. \& FROST, C.D. (2008): A Geochemical Classification for Feldspathic Igneous Rocks.- J Petrology, 49/11, 1955-1969. doi: 10.1093/petrology/egn054

FROST, B.R., BARNES, C.G., COLLINS, W.J., ARCULUS, R.J., ELLIS, D.J. \& FROST, C.D. (2001): A geochemical classification for granitic rocks.- J. Petrol., 42/11, 20332048. doi: 10.1093/petrology/42.11.2033

FROST, C.D. \& FROST, B.R. (2011): On Ferroan (A-type) Granitoids: Their Compositional Variability and Modes of Origin.- J. Petrol., 52/1,39-53. doi: 10.1093/petrology/egq070

GALLHOFER, D., VON QUADT, A., PEYTCHEVA, I., SCHMID, S.M. \& HEINRICH, C.A. (2015): Tectonic, magmatic, and metallogenic evolution of the Late Cretaceous arc in the Carpathian-Balkan orogen.- Tectonics, 34/9, 1-24. doi: 10.1002/2015TC003834

HAMMASTROM, J.M. \& ZEN, E. (1986): Aluminium in hornblende: An empirical igneous geobarometer--Am. Mineral., 71, 1297-1313. 
HANSON G.N. (1978): The application of trace elements to the petrogenesis of igneous rocks of granitic composition.- Earth Planet. Sci. Lett., 38/1, 26-43. doi: 10.1016/0012-821X(78)90124-3

HOLLISTER, S., GRISSOM, G.C., PETERS, E.K.M., STOWELL, H.H. \& SISSON, V.B (1987): Confirmation of the empirical correlation of $\mathrm{Al}$ in hornblende with pressure of solidification of calc-alkaline plutons.-Am. Mineral., 72, 231-239.

HORVAT, M., KLÖTZLI, U., JAMIČIĆ, D., BUDA, G., KLÖTZLI, E. \& HAUZENBERGER, C. (2018): Geochronology of granitoids from Psunj and Papuk Mts., Croatia.- Geochronometria, 45/1, 198-210. doi: 10.1515/geochr-2015-0099

JANOUŠEK, V., FARROW, C.M. \& ERBAN, V. (2006): Interpretation of whole-rock geochemical data in igneous geochemistry: introducing Geochemical Data Toolkit (GCDkit).- J. Petrol., 47, 1255-1259. doi: 10.1093/petrology/eg1013

JOHNSON, M.C. \& RUTHERFORD, M.J. (1989): Experimental calibration od the aluminium-in-hornblende geobarometer with application to Long Valley caldera (California).- Geology, 17, 837-841.

LE BAS, M.J., LE MAITRE, R.W., STRECKEISEN, A. \& ZANETTIN, B. (1986): A chemical classification of volcanic rocks based on the total alkali-silica diagram.- J Petrology, 27, 745-750.

LEAKE, B.E., WOOLLEY, A.R., ARPS, C.E.S., BIRCH, W.D., GILBERT, M.C., GRICE, J.D., HAWTHORNE, F.C., KATO, A., KISCH, H.J., KRIVOVICHIEV, V.G., LINTHOUT, K., LAIRD, J., MARESCH, W.V., NICKEL, E.H., ROCK, N.M.S., SCHUMACHER, J.C., SMITH, D.C., STEPHENSON, N.C., UNGARETTI, L., WHITTAKER, E.J.W. \& YOUZHI, G. (1997): Nomenclature of amphiboles: report of the Subcommittee on amphiboles of the International Mineralogical Association, Commission on New Minerals and Mineral Names.- Can. Mineral., 35, 219-246.

LOISELLE, M.C. \& WONES, D.R. (1979): Characteristics and origin of anorogenic granites.- Geol. Soc. Am. Abstr. Programs, 11, p. 468.

LOWENSTERN, J.B., CLYNNE, M.A., \& BULLEN, T.D. (1997): Comagmatic A-Type Granophyre and Rhyolite from the Alid Volcanic Center, Eritrea, Northeast Africa.Journal of Petrology, 38, 1707-1721. doi: 10.1093/petroj/38.12.1707

MCDONOUGH, W.F. \& SUN, S.S. (1995): The composition of the Earth.- Chem. Geol., 120, 223-253. doi: 10.1016/0009-2541(94)00140-4

MILLER, C.F., MESCHTER MCDOWELL, S. \& MAPES, R.W. (2003): Hot and cold granites? Implications of zircon saturation temperatures and preservation of inheritance.- Geology, 31, 529-532. doi: 10.1130/0091-7613(2003)031\%3C0529:HAC GIO $\% 3 \mathrm{E} 2.0 . \mathrm{CO} ; 2$

MUTCH, E.J.F., BLUNDY, J.D., TATTITCH, B.C., COOPER, F.J. \& BROOKER, R.A. (2016): An experimental study of amphibole stability in low pressure granitic magmas and a revised $\mathrm{Al}$ in hornblende geobarometer.-Contrib. Mineral. Petrol., 171, 85. doi: 10.1007/s00410-016-1298-9

NACHIT, H., RAZAFIMAHEFA, N., STUSSI, J.M. \& CARRON, J.P. (1985): Composition chimique des biotites et typologie magmatique des granitoides [Chemical compositiona of biotite and magmatic typology of granites - in French].- C.R. Academic Sciences Paris, Series 2, 301, 813-818

PAMIĆ, J. (2002): The Sava-Vardar Zone of the Dinarides and Hellenides versus the Vardar Ocean.- Eclogae Geol. Helv., 95, 99-113.

PAMIĆ, J. (1999): Kristalina podloga južnih dijelova Panonskog bazena - temeljena na površinskim i bušotinskim podacima [Crystalline basement of the South Pannonian Basin based on surface and subsurface data - in Croatian].- Nafta, 50, 291-310.

PAMIĆ, J. (1987): Mladoalpinski alkalijsko-feldspatski graniti (aljaskiti) Požeške gore u Slavoniji [Young-Alpine alkali feldspar granites (alaskites) from Mt. Požeška Gora in Slavonia, northern Yugoslavia - in Croatian].- Geologija, 30, 183-205.

PAMIĆ, J. (1986): Magmatic and metamorphic complexes of the adjoining area of the northernmost Dinarides and Pannonian Mass.-Acta. Geol. Hung., 29, 203-220.

PAMIĆ, J., BELAK, M., BULLEN, T.D., LANPHERE, M.A. \& MCKEE, E.H. (2000): Geochemistry and geodynamics of a Late Cretaceous bimodal volcanic association from the southern part of the Pannonian Basin in Slavonia (Northern Croatia).- Mineral. Petrol., 68, 271-296.

PAMIĆ, J., LANPHERE, M. \& BELAK, M. (1996): Hercynian I-type and S-type granitoids from the Slavonian mountains (southern Pannonian Basin, northern Croatia).N. Jb. Miner. Abh., 171, 155-186.

PAMIĆ, J. \& PÉCSKAY, Z. (1994): Geochronology of Upper Cretaceous and Tertiary igneous rocks from the Slavonia-Srijem Depression (Southern Pannonian Basin) and their basic petrological features.- Nafta, 45, 331-339.

PAMIĆ, J. \& LANPHERE, M. (1991): Hercinske granitne i metamorfne stijene Papuka, Psunja, Krndije i okolne podloge Panonskog bazena u Slavoniji (sjeverna Hrvatska, Jugoslavija) [Hercynian Granites and Metamorphic Rocks from The Mts. Papuk, Psunj, Krndija, and The Surrounding Basement of The Pannonian Basin in Slavonija (Northern Croatia, Yugoslavia) - in Croatian].-- Geologija, 34, 81-253.

PAMIĆ, J., INJUK, J. \& JAKŠIĆ, M. (1988/1989): Prilog geokemijskom poznavanju gornjokredne bimodalne vulkanske asocijacije Požeške gore u Slavoniji (sjeverna Hrvatska, Jugoslavija) [Some geochemical features of the Upper Cretaceous bimodal volcanic association from the Požeška Gore Mt. in Slavonija (northern Croatia, Yugoslavia) - in Croatian].- Geologija, 31, 415-435.

PAMIĆ, J. \& INJUK, J. (1988): Alpinske granitoidne stijene planine Prosare u sjevernoj Bosni [Alpine granitoid rocks of Prosara Mt. in northern Bosnia, Yugoslavia - in
Croatian].- In: SLIŠKOVIĆ, T. (ed): Zbornik referata naučnog skupa „Minerali, stijene, izumrli i živi svijet BiH“,Zemaljski muzej Bosne i Hercegovine, 93-103.

PAMIĆ, J., LANPHERE, M. \& MCKEE, E. (1988): Radiometric ages of metamorphic and associated igneous rocks of the Slavonian Mountains in the southern part of the Pannonian Basin, Yugoslavia.-Acta Geologica, 18, 13-39.

PAPOUTSA, A., PE-PIPER, G. \& PIPER, D.J.W. (2015): Systematic mineralogical diversity in A-type granitic intrusions: Control of magmatic source and geological processes.- Geol. Soc. Am. Bull. doi:10.1130/B31245

PEARCE, J.A., HARRIS, N.B.W. \& TINDLE, A.G. (1984): Trace element discrimination diagrams for the tectonic interpretation of granitic rocks.- J. Petrol., 25/4, 956-983. doi: 10.1093/petrology/25.4.956

PECERILLO, A. \& TAYLOR, S.R. (1976): Geochemistry of Eocene Calc Alkaline Volcanic Rocks from Kastamonu Area, Northern Turkey.- Contrib. to Mineral. Petrol., 58, 63-81.

PRELEVIĆ, D., WEHRHEIM, S., REUTTER, M., ROMER, R.L., BOEV, B., BOŽOVIĆ, M., VAN DEN BOGAARD, P., CVETKOVIĆ, V. \& SCHMID, S.M. (2017): The Late Cretaceous Klepa basalts in Macedonia (FYROM) - Constraints on the final stage of Tethys closure in the Balkans.- Terra Nova, 29, 145-153. doi: 10.1111/ ter. 12264

ROLLINSON, H. \& PEASE, V. (2021): Using geochemical data: to understand geological properties (2nd ed.).- Cambridge: Cambridge University Press, 358 p.

SCHAEN, A.J., COTTLE, J.M., SINGER, B.S., KELLER, C.B., GARIBALDI, N. \& SCHOENE, B. (2017): Complementary crystal accumulation and rhyolite melt segregation in a late Miocene Andean pluton.- Geology, 45/9, 835-838. doi: 10.1130/ G39167.1

SCHMID, S.M., FÜGENSCHUH, B., KOUNOV, A., MATENCO, L., NIEVERGELT, P., OBERHÄNSLI, R., PLEUGER, J., SCHEFER, S., SCHUSTER, R., TOMLJENOVIĆ, B., USTASZEWSKI, K. \& VAN HINSENBERG, D.J.J. (2020): Tectonic units of the Alpine collision zone between Eastern Alps and western Turkey.- Gondwana Res., 78, 308-374. doi: 10.1016/j.gr.2019.07.005

SCHMID, S.M., BERNOULLI, D., FÜGENSCHUH, B., MATENCO, L., SCHEFER, S., SCHUSTER, R., TISCHLER, M. \& USTASZEWSKI, K. (2008): The Alpine-Carpathian-Dinaridic orogenic system: Correlation and evolution of tectonic units.Swiss J. Geosci., 101, 139-183. doi: 10.1007/s00015-008-1247-3

SCHMIDT, M.W. (1992): Amphibole composition in tonalite as a function of pressure: An experimental calibration of the Al-in-hornblende barometer-- Contrib. to Mineral. Petrol., 110/2-3, 266-268. Doi: 10.1007/BF00310745

SOKOL, K., PRELEVIĆ, D., ROMER, R.L., BOŽOVIĆ, M., VAN DER BOGAARD, P., STEFANOVA, E., KOSTIĆ, B. \& ČOKULOV, N. (2020): Cretaceous ultrapotassic magmatism from the Sava-Vardar Zone of the Balkans.- Lithos, 354-355. doi: 10.1016/j.lithos.2019.105268

SUN, S.S. \& MCDONOUGH, W.E. (1989): Chemical and isotopic systematics of oceanic basalts: implications for mantle composition and processes.- In: SAUNDERS, A.D. \& NORRY, M.J. (eds). Magmatism in the Ocean Basins, Saunders, Geological Society, London, Special Publications, 42, 313-345. doi: 10.1144/GSL. SP.1989.042.01.19

STARIJAŠ, B., GERDES, A., BALEN, D., TIBLJAŠ, D. \& FINGER, F. (2010): The Moslavačka Gora crystalline massif in Croatia: a Cretaceous heat dome within remnant Ordovician granitoid crust.- Swiss. J. Geosci., 103/1, 62-82. doi: 10.1007/ s00015-010-0007-3

STRECKEISEN, A. (1974): Classification and nomenclature of plutonic rocks. Geol. Rundsch., 63, 773-786.

ŠPARICA, M. \& PAMIĆ, J. (1986): Prilog poznavanju tektonike Požeške gore u Slavoniji. [Contribution to the knowledge of tectonics of Požeška Gora in Slavonia - in Croatian].- Rad JAZU, 424, 85-96.

USTASZEWSKI, K., KOUNOV, A., SCHMID, S.M., SCHALTEGGER, U., KRENN, E., FRANK, W. \& FÜGENSCHUH, B. (2010): Evolution of the Adria-Europe plate boundary in the northern Dinarides: From continent-continent collision to back-arc extension.- Tectonics, 29/6. doi: 10.1029/2010TC002668

USTASZEWSKI, K., SCHMID, S.M., LUGOVIĆ, B., SCHALTEGGER, U., BERNOULLI, D., HOTTINGER, L., KOUNOV, A., FÜGENSCHUH, B. \& SCHEFER, S. (2009): Late Cretaceous intra-oceanic magmatism in the internal Dinarides (northern Bosnia and Herzegovina): Implications for the collision of the Adriatic and European plates.- Lithos, 108/1-4, 106-125. doi: 10.1016/j.lithos.2008.09.010

VERNON, R.H. (2008): A Practical Guide to Rock Microstructure-- Cambridge University Press, Cambridge, $606 \mathrm{p}$

WATSON, E.B. \& HARRISON, T.M. (1983): Zircon saturation revisited: Temperature and composition effects in a variety of crustal magma types.- Earth Planet. Sci. Lett., 64, 295-304. doi: 10.1016/0012-821X(83)90211-X

WHALEN, J.B., CURRIE, K.L., CHAPPELL, B.W. (1987): A-type granites: geochemical characteristics, discrimination and petrogenesis.- Contrib. to Mineral. Petrol., 95, 407-419. doi: 10.1007/BF00402202

WERE, P. \& KEPPLER, H (2021): Trace element fractionation between biotite, allanite, and granitic melt.- Contrib. Mineral. Petrol, 176/9, 74. doi: 10.1007/s00410-02101831-3

WHITNEY, D.L. \& EVANS, B.W. (2010): Abbreviations for names of rock-forming minerals.-Am. Mineral., 95/1, 185-187. doi: 10.2138/am.2010.3371 